\title{
Metabonomics Combined with UPLC-MS Chemical Profile for Discovery of Antidepressant Ingredients of a Traditional Chinese Medicines Formula, Chaihu-Shu-Gan-San
}

\author{
Hongmei Jia, ${ }^{1}$ Zhiheng Su, ${ }^{2}$ Wei Long, ${ }^{3}$ Yuetao Liu, ${ }^{1}$ Xing Chang, ${ }^{1}$ Hongwu Zhang, \\ Gang Ding, ${ }^{1}$ Yufei Feng, ${ }^{4}$ Dayong Cai, ${ }^{1}$ and Zhongmei Zou ${ }^{1}$ \\ ${ }^{1}$ Institute of Medicinal Plant Development, Chinese Academy of Medical Sciences and Peking Union Medical College, \\ Beijing 100193, China \\ ${ }^{2}$ School of Pharmaceutical Science, Guangxi Medical University, Nanning 530021, China \\ ${ }^{3}$ Tianjin Key Laboratory of Molecular Nuclear Medicine, Institute of Radiation Medicine, Chinese Academy of Medical Science, \\ Tianjin 300192, China \\ ${ }^{4}$ Department of Pharmacy, Beijing Hospital, Ministry of Public Health, Beijing 100730, China
}

Correspondence should be addressed to Zhongmei Zou; zmzou@implad.ac.cn

Received 21 November 2012; Revised 15 February 2013; Accepted 18 February 2013

Academic Editor: Adair Roberto Soares Santos

Copyright (C) 2013 Hongmei Jia et al. This is an open access article distributed under the Creative Commons Attribution License, which permits unrestricted use, distribution, and reproduction in any medium, provided the original work is properly cited.

\begin{abstract}
This study proposed a new strategy for uncovering the active chemical constituents of a traditional Chinese medicines (TCMs) formula, Chaihu-Shu-Gan-San (CSGS). Metabonomics and chemical profile were integrated in combination with the multivariate statistical analysis (MVA) to discover the chemical constituents which contribute to the antidepressant effect of CSGS. Based upon the difference between CSGS and QZ (CSGS without Zhi-Qiao) extracts in the chemical profiles and the regulations of metabolic disturbances induced by CUMS, synephrine, naringin, hesperidin, and neohesperidin were recognized as the active constituents of CSGS from Zhi-qiao responsible for those missing regulations of CSGS when Zhi-Qiao was subtracted from the whole formula. They participated in the regulations of the deviated metabolites 2-4, 10-14, and 22-25, involved in metabolic pathways of ketone bodies synthesis, phenylalanine, tyrosine and tryptophan biosynthesis, valine, aspartate, glutamate metabolism, and glycolysis/gluconeogenesis. Furthermore, the assay of MAO-A activity confirmed the potential antidepressant effect of naringin and its active sites on the MAO-A was inferred by molecular docking study. The integration of metabonomics and chemical profile was proved to be a useful strategy for uncovering what the active chemical constituents in TCM formula are and how they make contributions for the efficacy of the formula.
\end{abstract}

\section{Introduction}

Traditional Chinese medicines (TCMs) have been widely used in many oriental countries for thousands of years [1] and received widespread acceptance and attention due to their reliable therapeutic efficacy with low side effects [2]. It is universally acknowledged that the holistic and dynamic effects were achieved by multitargets interactions of the multiconstituents in TCMs. However, the complexity and interaction of multiconstituents in TCMs make the identification of the chemical constituents related to the efficacy and the definition of their mechanism of action challenging.
Novel approaches are in great demand to provide deeper insight into the correlation of chemical constituents with efficacy of herbal formula.

Chromatographic fingerprinting has been internationally accepted as an efficient technique for direct identification of multicomponents and quality control of TCMs [3]. Due to the wide suitability, high sensitivity, and sufficient structural information, liquid chromatography coupled with electrospray ionization tandem mass spectrometry (LC-ESI-MS ${ }^{\mathrm{n}}$ ) has become more and more popular for investigation of herbal medicines [4]. Although on-line qualitative and quantitative analysis of chemical constituents in TCM formulas 


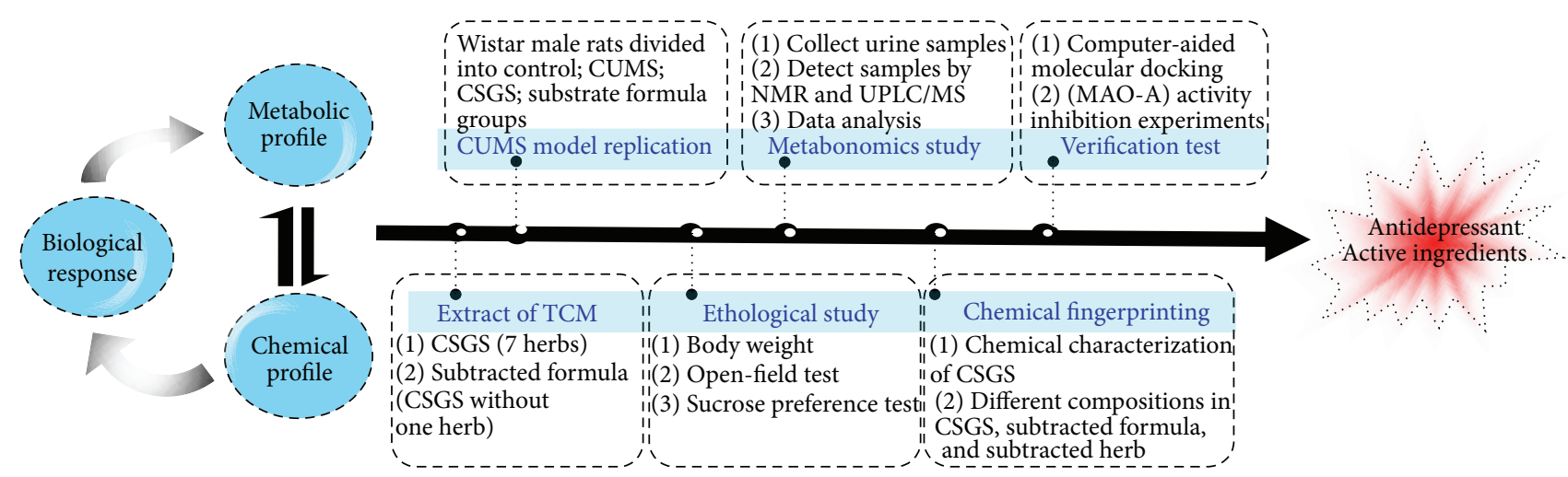

FIGURE 1: The proposed strategy for discovering the active constituents of CSGS responsible for its therapeutic effect on depression.

by LC-MS/MS was powerful for quality control, chromatographic profile fails to discern the correlation between the identified compounds and efficacy.

Metabonomics is the comparative analysis of metabolites and their dynamic flux associated with the response of living systems to pathophysiological stimuli or genetic modification [5]. Based on the global metabolic profile in biological samples such as urine, plasma, and tissue [2], it provides variation of the whole metabolic networks for characterizing pathological states in animals and humans, as well giving diagnostic information and presenting mechanistic insight into the biochemical effects of the toxins and drugs $[6,7]$. In agreement with the holistic thinking of TCM, metabonomics has shown potential in evaluation of therapeutic effect of TCMs [8] and may provide the links needed for the complex metabolite mixtures in TCMs and molecular pharmacology [9].

Chaihu-Shu-Gan-San (CSGS) is one of the most widely used TCM formulas for treatment of depression clinically in China [10]. It is composed of seven Chinese herb medicines, that is, the roots of Bupleurum chinense DC (Chai-Hu), the pulp of Citrus reticulata Blanco (Chen-Pi), the roots of Paeonia lactiflora Pall (Bai-Shao), the pericarp of Citrus aurantium L. (Zhi-Qiao), the roots of Cyperus rotundus L. (Xiang-Fu), the roots of Ligusticum chuanxiong Hort (Chuan-Xiong), and the roots of Glycyrrhiza uralensis Fisch (Gan-Cao). The metabonomics study suggested that the antidepressant effect of CSGS could involve in regulating the dysfunctions of multiple metabolic pathways [11]. And the chemical constituents in CSGS were identified by LCMS/MS and its antioxidant constituents were profiled by combination of 96-well plate collection of elutes from HPLC analysis and microplate spectrophotometer [12]. However, the contributions of chemical constituents in CSGS to its antidepressant effect are still not clear.

Here, a new integrated strategy of metabonomics and chemical profile in combination with the multivariate statistical analysis (MVA) was proposed to discover which of the chemical constituents in CSGS were responsible for its therapeutic effect (Figure 1). One of the key herbs in CSGS, Zhi-Qiao, was used as an example to explore the antidepressant chemical constituents from single herb in CSGS. The regulations of CSGS and QZ (CSGS without Zhi-Qiao) in metabolic disturbance induced by chronic unpredicted mild stress (CUMS) were explored by NMR and LC-MSbased metabonomics. Those regulations missed in QZ treated group should be related to the constituents existing in ZhiQiao but missed in QZ compared with CSGS. The chemical profiles of CSGS and QZ extracts through LC-MS/MS analysis were subjected to multivariate statistics analysis and those constituents that made contributions to discriminate the two extracts were considered as potential active constituents of CSGS from Zhi-Qiao, responsible for those effects that disappeared in QZ. Finally, combination of the results from metabonomics and chemical profile could tell us the contributions of Zhi-Qiao to CSGS both in chemical constituents and in regulations of metabolic pathways.

\section{Materials and Methods}

2.1. Chemicals and Reagents. HPLC-grade acetonitrile was purchased from Merck (Darmstadt, Germany). The water used for UPLC was purified by a Milli-Q system (Millipore, France). Formic acid (HPLC grade) was purchased from Tedia (Fairfield, USA). Pargyline, sodium phosphate, and sucrose were purchased from Sigma-Aldrich (St. Louis, MO, USA). Commercial kits used for determining MAO activity were obtained from the Jiancheng Institute of Biotechnology (Nanjing, China). Synephrine, hesperidin, neohesperidin and naringin were purchased from Tongtian Biotechnology Co., Ltd. (Shanghai, China). Fluoxetine hydrochloride was purchased from Eli Lilly and Company (Suzhou, China).

All raw herbal medicines were purchased from Beijing Tongren Tang Pharmaceutical Co. Ltd. (Beijing, China) and kept in our laboratory at Institute of Medicinal Plant Development, Chinese Academy of Medical Sciences, and Peking Union Medical College, China. The mixed crude herbs, Chai-Hu, Chen-Pi, Bai-Shao, Zhi-Qiao, Xiang-Fu, ChuanXiong, and Gan-Cao in the proportions of $4: 4: 3: 3: 3: 3: 1$ by weight, were crushed into small pieces. The CSGS extract was prepared based on the traditional method as previously described [10]. The yield of the CSGS extract was $18.87 \%$. The Zhi-Qiao (yield: 20.28\%) and QZ (all herbs except Zhi-Qiao, yield: $20.00 \%$ ) were prepared using procedures identical to that for CSGS. 
2.2. Rats and Treatment. 40 healthy, adult, male Wistar rats, weighing $200 \pm 20 \mathrm{~g}$ each, were purchased from the Institute of Laboratory Animal Science, CAMS and PUMC (Beijing, China). The rats were housed individually in cages for one week to adapt to the environment under controlled conditions of $12 \mathrm{~h}$ light-12 h dark cycles (lights on from 6:00 a.m. to $6: 00$ p.m.), $10 \%$ relative humidity, and temperature $(20 \pm$ $\left.3^{\circ} \mathrm{C}\right)$ with commercial diet and water available ad libitum. All experimental procedures were approved by the Ethics Committee of the Institute of Medicinal Plant Development, CAMS \& PUMC.

The animals were randomly divided into 5 groups: (1) control group, (2) CUMS group, (3) positive control group, (4) CSGS treated group, and (5) QZ treated group. All rats except control group were subjected to a series of variable stimuli as previously described [10] after minor modification. The rats in the positive control, CSGS, and QZ treated groups were administrated with fluoxetine $(3.0 \mathrm{mg} / \mathrm{kg})$ and extracts of CSGS (equivalent to $31.5 \mathrm{~g}$ crude drug/ $\mathrm{kg}$ body weight) and QZ (equivalent to $27.0 \mathrm{~g}$ crude drug $/ \mathrm{kg}$ body weight), respectively, for 28 consecutive days, and the rats were sacrificed on the 28 th day.

2.3. Sample Collection and Preparation. All rats were housed in metabolic cages ( 1 per cage) so that the $24 \mathrm{~h}$ urine samples could be collected in collection bottles containing $\mathrm{NaN}_{3}$ $(0.05 \% \mathrm{wt} / \mathrm{vol})$ on the $28 \mathrm{th}$ day. 16 urine samples were randomly selected from the urine samples of each group, and $1 \mathrm{~mL}$ urine was taken from each sample, respectively; then the $16 \mathrm{~mL}$ urine was mixed together as the QC sample. The QC sample was used for the optimization of UPLC-Q-TOF/MS conditions. Every day, the stability of the instrument was tested with QC sample in order to make sure that the instrument was in the same condition during the whole analytical procedure. The urine samples were centrifuged $(5000 \mathrm{rpm}$ for $10 \mathrm{~min}, 4^{\circ} \mathrm{C}$ ), and the supernatants were divided into two aliquots and stored at $-80^{\circ} \mathrm{C}$ before analysis. One aliquot was used for NMR analysis and the other for UPLC/MS analysis.

2.4. ${ }^{1}$ H NMR Spectroscopic Measurement of Urine Samples. An aliquot of $400 \mu \mathrm{L}$ urine was thawed at room temperature and mixed with $200 \mu \mathrm{L}$ of phosphate buffer $(0.2 \mathrm{M}$ $\mathrm{Na}_{2} \mathrm{HPO}_{4}$ and $0.2 \mathrm{M} \mathrm{NaH} \mathrm{PO}_{4}$ in $\mathrm{D}_{2} \mathrm{O}$ containing $0.05 \%$ wt/vol 3-trimethylsilyl-(2,2,3,3- $\left.{ }^{2} \mathrm{H}_{4}\right)$-1-propionate (TSP); $\mathrm{pH}$ 7.4). Phosphate buffer minimized chemical shift variation because of different $\mathrm{pH}$ in urine samples, with $\mathrm{D}_{2} \mathrm{O}$ as a field lock and TSP as a chemical shift reference. The mixture was centrifuged (13000 rpm, $15 \mathrm{~min})$ and the supernatant $(550 \mu \mathrm{L})$ of each sample was then transferred into a $5 \mathrm{~mm}$ o.d. NMR tube individually. All ${ }^{1} \mathrm{H}$ NMR spectra were recorded at $300 \mathrm{~K}$ on a Bruker AV III 600 spectrometer (Bruker Biospin, Germany) equipped with an inverse $5 \mathrm{~mm}$ Bruker probe operating at $600.13 \mathrm{MHz}{ }^{1} \mathrm{H}$ frequency. ${ }^{1} \mathrm{H}$ NMR spectra were acquired using water-suppressed NOSEYGPPR1D (RD-90- $t$ $\left.90-t_{m}-90-A C Q\right)$. The water signal suppression was achieved with weak irradiation on the water peak during the recycling delay $(\mathrm{RD}=4.0 \mathrm{~s})$ and mixing time $\left(t_{m}=0.10 \mathrm{~s}\right)$. The $90^{\circ}$ pulse length was adjusted to $\sim 10 \mu \mathrm{s}$; a total of 128 transients were collected into $96 \mathrm{~K}$ data points over a spectral width of $20 \mathrm{ppm}$ with an acquisition time of $3.07 \mathrm{~s}$.

Prior to Fourier transformation, the FIDs for one-dimensional data were multiplied by an exponential function equivalent to a line broadening factor of $0.5 \mathrm{~Hz}$ and were zero-filled to $128 \mathrm{k}$. All NMR spectra were then corrected for phase and baseline distortions using Topspin software (v2.1, Bruker-Biospin, Germany). ${ }^{1} \mathrm{H}$ NMR chemical shifts in the spectra were referenced to TSP at $\delta 0.00$. All ${ }^{1} \mathrm{H}$ NMR spectra from urine samples were data-reduced to integrated regions $0.004 \mathrm{ppm}$ wide corresponding to the region $\delta 0.5-9.5 \mathrm{using}$ AMIX software package (v3.9.2, Bruker Biospin, Germany). The regions $\delta$ 4.67-5.10 were removed to avoid the effect of residual water saturation. Each NMR data set was binned to 1875 variables to minimize the effects of $\mathrm{pH}$ and ionic concentrations.

2.5. UPLC/MS Measurement of Urine Samples. All urine samples were thawed at room temperature before analysis and centrifuged at $13,000 \mathrm{rpm}$ for $10 \mathrm{~min}$ at $4^{\circ} \mathrm{C}$. The supernatant was diluted at a ratio of $1: 1$ with water and an aliquot of $5 \mu \mathrm{L}$ was injected for UPLC-Q-TOF/MS analysis after filtration through a $0.22 \mu \mathrm{M}$ membrane filter.

The urine samples were analyzed on Waters Acquity Ultra Performance LC system (Waters Corporation, Milford, MA, USA) equipped with a BEH C18 column $(100 \mathrm{~mm} \times$ $2.1 \mathrm{~mm}, 1.7 \mu \mathrm{m})$. The mobile phase was composed of water (A) and acetonitrile (B) each containing $0.1 \%$ formic acid. A solvent gradient system was used for detecting the urine samples: $1 \% \mathrm{~B}$ from 0 to $1 \mathrm{~min}, 1-32 \% \mathrm{~B}$ from 1 to $9 \mathrm{~min}$, $32-99 \%$ B from 9 to $11 \mathrm{~min}$, and $99 \%$ B from $12-15 \mathrm{~min}$. The flow rate was $0.45 \mathrm{~mL} / \mathrm{min}$. All the samples were kept at $4^{\circ} \mathrm{C}$ during the analysis. The mass spectrometric data were collected using Q-TOF analyzer in SYNAPT HDMS system (Waters Corporation, Milford, MA, USA) in both positive and negative ion modes. Experimental parameters were set as previously described [11].

The raw data were analyzed using the MarkerLynx Applications Manager version 4.1 (Waters, Manchester, UK), which allowed deconvolution, alignment, and data reduction to give a list of retention time and mass pairs with corresponding intensities for all the detected peaks from each data file in the data set. The main parameters were set as follows: retention time range (RT) range $0.5-15 \mathrm{~min}$, mass range $50-1200 \mathrm{amu}$, mass tolerance 0.02 , minimum intensity $1 \%$, mass window 0.05 , retention time window 0.20 , and noise elimination level 6.

The extracted ion chromatographic peaks of ten ions in positive mode (RT_m/z: 1.31_98.0603, 1.47_297.1443, 1.73_126.0919, 3.01_105.0334, 3.17_372.2382, 3.68_91.0541, 4.43_130.0654, 5.67_170.0612, 5.93_243.1021 and 6.68 203.1108) and ten ions in negative mode (RT $m / z$ : 0.83_182.0461, 1.23_144.0665, 2.21_158.0828, 3.00_178.0511, 3.38_222.0793, 4.31_283.0832, 4.76_173.0824, 6.10_187.0972, 7.36_201.1133 and 7.86_319.1380) from QC sample were selected for method validation. The repeatability of method was evaluated using six replicates of QC sample in positive and negative ion modes, respectively. The relative standard 
deviations (R.S.D\%) of retention times and $m / z$ were $0-$ $0.4818 \%$ and $0.0002-0.0006 \%$ in positive mode and $0-$ $0.3753 \%$ and $0.0003-0.0008 \%$ in negative mode, respectively. Precision of injection was carried out by six replicated analyses of the same urine sample. The relative standard deviations (R.S.D\%) of retention times and $m / z$ were $0-0.3719 \%$ and $0.0002-0.0007 \%$ in positive mode and $0.09-$ $0.4173 \%$ and $0.0002-0.0009 \%$ in negative mode, respectively.

2.6. UPLC-MS/MS Analysis of the Extracts of CSGS, QZ, and Zhi-Qiao. $50 \mathrm{mg}$ of CSGS extract (equivalent to $264.97 \mathrm{mg}$ of raw herbs in the proportions listed above) was dissolved in $5 \mathrm{~mL}$ of deionized water. The solution was filtered through a $0.22 \mu \mathrm{m}$ filter membrane and $5 \mu \mathrm{L}$ of resulting solution was injected into the UPLC system for UPLCMS/MS analysis. For analysis of Zhi-Qiao (7.40 mg) and QZ extracts $(45.53 \mathrm{mg})$, an amount of Zhi-Qiao or QZ extract representing the same amount of raw herb equivalents in the $50 \mathrm{mg}$ of CSGS extract was prepared and analyzed identically to CSGS.

Chromatographic separation was performed on an Acquity UPLC BEH C18 column $(2.1 \times 100 \mathrm{~mm}, 1.7 \mu \mathrm{m}$, Waters Corp., Milford, USA) using an ACQUITYTM UPLC system (Waters), equipped with a binary solvent delivery system, an autosampler with $4^{\circ} \mathrm{C}$, and a PDA detector. The column was maintained at $40^{\circ} \mathrm{C}$ and eluted at a flow rate of $0.45 \mathrm{~mL} / \mathrm{min}$, using a mobile phase of (A) $0.1 \%$ (by volume) formic acid in water and (B) acetonitrile. The gradient program was optimized as follows: $0-2 \mathrm{~min}, 5 \% \mathrm{~B}$ to $10 \% \mathrm{~B}$; $2-8 \mathrm{~min}, 10 \% \mathrm{~B}$ to $20 \% \mathrm{~B} ; 8-10 \mathrm{~min}, 20 \% \mathrm{~B}$ to $40 \% \mathrm{~B} ; 10-$ $13 \mathrm{~min}, 40 \% \mathrm{~B}$ to $90 \% \mathrm{~B} ; 13-15 \mathrm{~min}$ and $90 \% \mathrm{~B}$ to $99 \% \mathrm{~B}$; 16-20 min, equilibration with $5 \% \mathrm{~B}$. The mass spectrometric data was collected using Q-TOF analyzer in SYNAPT HDMS system (Waters Corporation, Milford, MA, USA) in both positive and negative ion modes. The source temperature was set at $120^{\circ} \mathrm{C}$ with a cone gas flow of $30 \mathrm{~L} / \mathrm{h}$, a desolvation gas temperature of $450^{\circ} \mathrm{C}$ with a desolation gas flow of $800 \mathrm{~L} / \mathrm{h}$. The capillary voltage was set to $3.0 \mathrm{kV}$ and $2.5 \mathrm{kV}$ for positive and negative ion modes, respectively, and the cone voltage was set to $35 \mathrm{~V}$. Centroid data was collected from $\mathrm{m} / z 50$ to 1200 with a scan time of $0.3 \mathrm{~s}$ and interscan delay of $0.02 \mathrm{~s}$ over a $15 \mathrm{~min}$ analysis time. Leucine-enkephalin was used as the lock mass $(\mathrm{m} / z 556.2771$ in positive mode and $\mathrm{m} / z 554.2615$ in negative mode) at a concentration of $0.5 \mu \mathrm{g} / \mathrm{mL}$ with a flow rate of $80 \mu \mathrm{L} / \mathrm{min}$. The lock spray frequency was set at $20 \mathrm{~s}$.

2.7. Monoamine Oxidase A (MAO-A) Activity Assay. MAO was purified from the rat liver according to the Gómez method [13] after minor modification. The liver tissue was rapidly removed into ice cold homogenized $(1: 10, \mathrm{w} / \mathrm{v})$ in $50 \mathrm{mM} \mathrm{KH}_{2} \mathrm{PO}_{4}$ buffer, $\mathrm{pH} 7.2$, containing $0.25 \mathrm{mM}$ sucrose. Following centrifugation at $1000 \mathrm{~g}$ for $10 \mathrm{~min}$, the supernatant was centrifuged at $10,000 \mathrm{~g}$ for $30 \mathrm{~min}$ to obtain crude mitochondrial pellet.

The MAO-A activity was measured by spectrophotometrically (Mapada, UV-3100, China) using enzymatic kits according to the instructions (Nanjing Jiancheng Institute of Biotechnology). $\mathrm{IC}_{50}$ values were used to estimate the inhibition calculated in relation to a sample of the enzyme treated under the same conditions without inhibitor, versus inhibitor concentration.

2.8. Computer-Aided Molecular Docking Experiments. Molecular docking of naringenin into the active sites of MAOA was carried out by using the Windows based software package-Molecular Operating Environment (MOE, Version 2008.10, Chemical Computing Group, Canada). The ligands to be docked were constructed in Discovery Studio (DS, Version 2.5.5, Accelrys Software Inc. USA) and the hydrogen atoms were added according to the appropriate protonation states. Since flexible ligand docking was employed, its geometries required only brief optimization using a fast Dreiding-like force field (1000 iterations) in DS. During the process of optimization, the element, bond orders, number of bonds, and valence were taken into consideration when the terms of the energy equation were calculated. After geometry optimization, the final conformers of ligands were used as starting point for docking. The X-ray crystallographic structures of MAO-A in complex with harmine (PDB code: $2 \mathrm{Z} 5 \mathrm{X}$ ) were obtained from the Brookhaven Protein Data Bank (http://www.rcsb.org/pdb). Hydrogen atoms were added to the receptor models according to the appropriate protonation states of the ionizable amino acids at $\mathrm{pH} 7.4$ and the valences of the FAD cofactors (oxidized state) and cocrystallized ligands were corrected and hydrogen atoms were added, also according to the appropriate protonation state at $\mathrm{pH} 7.4$. Automated docking was subsequently carried out with the Docking Suit of MOE 2008. Total ligand flexibility was used in this protocol whereby the final ligand conformations were determined by the Monte Carlo conformation search method set to a variable number of trial runs. The docked ligands were further refined using in situ-ligand minimization with the Smart Minimizer algorithm. All parameters for the docking runs were set to their default values and ten possible binding solutions were computed for each docked ligand. The bestranked binding conformation of ligand was determined according to the Dock Score values. The illustrations were prepared with PyMOL [14].

2.9. Data Analysis. To diminish the deviation in data analysis from individual variance of urine samples, the data were normalized by a creatinine calibration method; that is, the metabolite intensity was divided by the creatinine concentration of each sample. Then according to the $80 \%$ rule $[15,16]$, only variables having more than $80 \%$ nonzero measurement values were kept in the peak list. Multivariate statistical analysis (MVA) and modeling were performed using Simca-p software (v12.0, Umetric, Umeå, Sweden). Import data were mean-centered and pareto-scaled prior to multivariate analysis. Principal components analysis (PCA) and orthogonal partial least squares discriminate analysis (OPLS-DA) were employed to process the acquired NMR and MS data. PCA was performed to discern the natural separation between different stages of samples by visual inspection of score plots. In the OPLS-DA model, samples from different groups were classified, and the results were visualized in the form of score plots to show the group 
clusters and S-plots to show the variables contribuing to the classification.

A two-tailed Student's $t$-test was performed using the Statistical Package for Social Science program (SPSS 16.0, SPSS, Chicago, IL, USA). The significance threshold was set at $P<0.05$ for this test.

\section{Results}

3.1. The Antidepressant Effects of CSGS and QZ Extracts on CUMS Treated Rats. The antidepressant effects of CSGS and QZ extracts were evaluated on a CUMS rat model, a well-validated animal model of depression (see supplementary material Table S1 available online at http://dx.doi.org/ $10.1155 / 2013 / 487158)$. The body weight, the number of horizontal movements, and the sucrose preference $[17,18]$ in the CUMS treated rats decreased significantly compared with control group. Rats treated with CSGS extract showed significant increases in the body weight, the number of horizontal movements, and the sucrose preference compared with CUMS group. Similar changes were observed in the QZ treated group except the number of horizontal movements in rats. The results indicated that QZ treated had similar therapeutic effect to CSGS in increasing body weight and sucrose preference, but did not improve the rat horizontal movement significantly compared with CSGS treated.

\subsection{Urinary Metabonomics Study by ${ }^{1} H$ NMR and UPLC-Q-TOF/MS}

3.2.1. Metabolic Profiles of CUMS-Induced Depression with CSGS and QZ Treatments. The urine samples collected on the 28th day from each group were analyzed by ${ }^{1} \mathrm{H}$ NMR (Figure S1 in supplementary material) and UPLC-Q-TOF/MS (Figures 2(A1), 2(B1), and 2(C1)). Principal components analysis (PCA) (Figures 2(A2), 2(B2), and 2(C2)) indicated that the metabolic profile of rat in CUMS model group deviated from the control, suggesting that significant biochemical changes were induced by CUMS. The metabolic profile of rats in CSGS treated group fairly differed from the CUMS group and was close to the control, indicating that the deviations induced by CUMS were significantly improved after treatment of CSGS. Similar results were observed in QZ treated group except that the mean center of spots in CSGS treated group was much closer to control group than that of QZ treated group, which was consistent with the results of ethological study.

\subsubsection{Potential Biomarkers in the CUMS-Induced Depression} Associated with Treatment of CSGS or QZ. OPLS-DA is a supervised multivariable statistical method to sharpen an already established separation between each two groups in PCA. In order to obtain better discrimination between CUMS and treatment groups, OPLS-DA was performed. As shown in Figures 2(A3), 2(B3), and 2(C3), the urine metabolic profiles of the control and CUMS groups, of the CSGS treated and CUMS groups, and of the QZ treated and CUMS groups were clearly separated.

According to the S-plot, those variables with lager VIP values (VIP $>1$ ) were selected as key metabolites for the differentiation between CUMS group and the control group, and the CSGS treated and CUMS groups, and the QZ treated and CUMS groups. If the metabolites not only represented the difference between CUMS group and the control group, but also the difference between CUMS group and CSGS or QZ treated group, they were considered as potential biomarkers of metabolic deviations in CUMS-induced depression mediated by CSGS or QZ (Figures 2(A4), 2(B4) and 2(C4)). As a result, twenty-eight variables were identified as potential biomarkers (Table 1 and Figure 3(a)). Fourteen of them were detected by ${ }^{1} \mathrm{H}$ NMR and the others were detected by UPLCQ-TOF/MS

Here, a potential biomarker with $m / z 154.1628$ at 1.07 min was taken as an example to illustrate the identification process. First, full-scan mass spectrum of the peak at $1.07 \mathrm{~min}$ (Figure S2A in supplementary material) in the positive ion mode provided a quasimolecular ion at $\mathrm{m} / z 154.1628$, suggesting a molecular formula with $\left[\mathrm{C}_{8} \mathrm{H}_{11} \mathrm{NO}_{2}\right]^{+}$(calculated to be 154.1789). Candidates were obtained in searching molecular weight at $154.1628 \mathrm{Da}$ (positive mode, MW tolerance $\pm 0.05 \mathrm{Da}$ ) from database including MassBank (http://www.massbank.jp/index.html), HMDB (http://www.hmdb.ca/), and METLIN (http://metlin.scripps .edu/). As a result, there are two candidates with molecular weight at $154.1628 \pm 0.05 \mathrm{Da}$, which are described as norphenylephrine and dopamine, respectively. The $\mathrm{MS}^{2}$ spectrum of the ion at $m / z 154.1628$ (Figure S2B in supplementary material) generated a series of ions at $m / z$ 137.1699, $119.1435,109.1635$ and 91.1547 , which are in agreement with the fragmentation pattern of dopamine [19] (Figure S3 supplementary material).

3.2.3. Difference between CSGS and QZ in Correction of Metabolic Deviations Induced by CUMS. The variation tendency of the identified potential biomarkers in CUMS model, CSGS treated, and QZ treated was shown in Table 1. The concentrations of sixteen metabolites $(\mathbf{1 - 4}, \mathbf{1 0 - 1 3}, \mathbf{1 9 - 2 6})$ were significantly decreased and twelve increased in CUMS group compared with normal control. Obviously, these metabolites were associated with depression-induced injury.

The deviations of all potential biomarkers induced by CUMS were corrected with CSGS treated except Nacetylserotonin (26), indole-3-ethanol (27), and 5-methoxytryptamine (28). The concentrations of fifteen metabolites $(1-4,10-13,19-25)$ were upregulated and the other ten metabolites $(\mathbf{5 - 9}, \mathbf{1 4}-\mathbf{1 8})$ were downregulated by CSGS, which represents the CSGS medication on chronic stress.

More importantly, QZ did not show any improvement on the deviations of nine variables (10-14, 22-25) (Figure 3) induced by CUMS, and the mediation effect of QZ on isoleucine (2), glutamate (3), and L-dopa (4) had no statistical significance as well. The results may explain why the metabolic pattern of rats in QZ treated group was not closer to the normal control group than that of CSGS treated group and suggested that the mediation effects of CSGS on the metabolites 2-4, 10-14, and 22-25 were lost in QZ. In other words, those mediations of CSGS should be provided by ZhiQiao. 


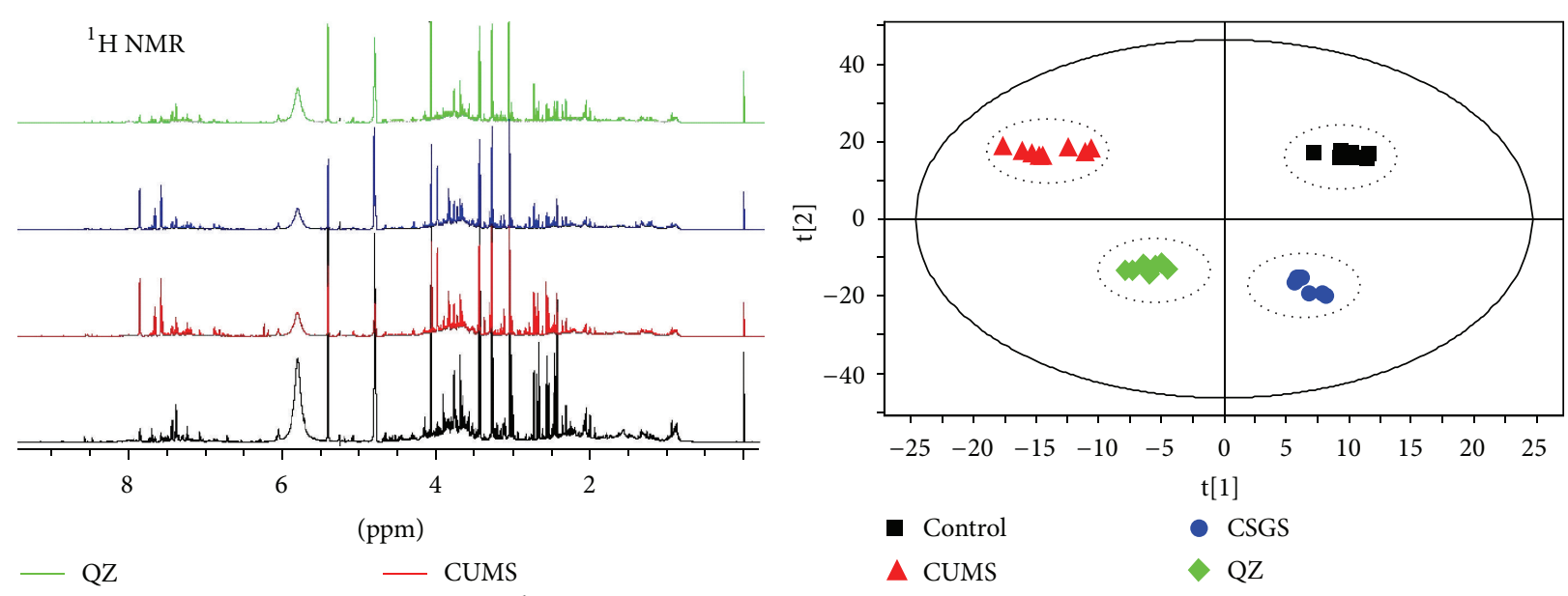

(A1)
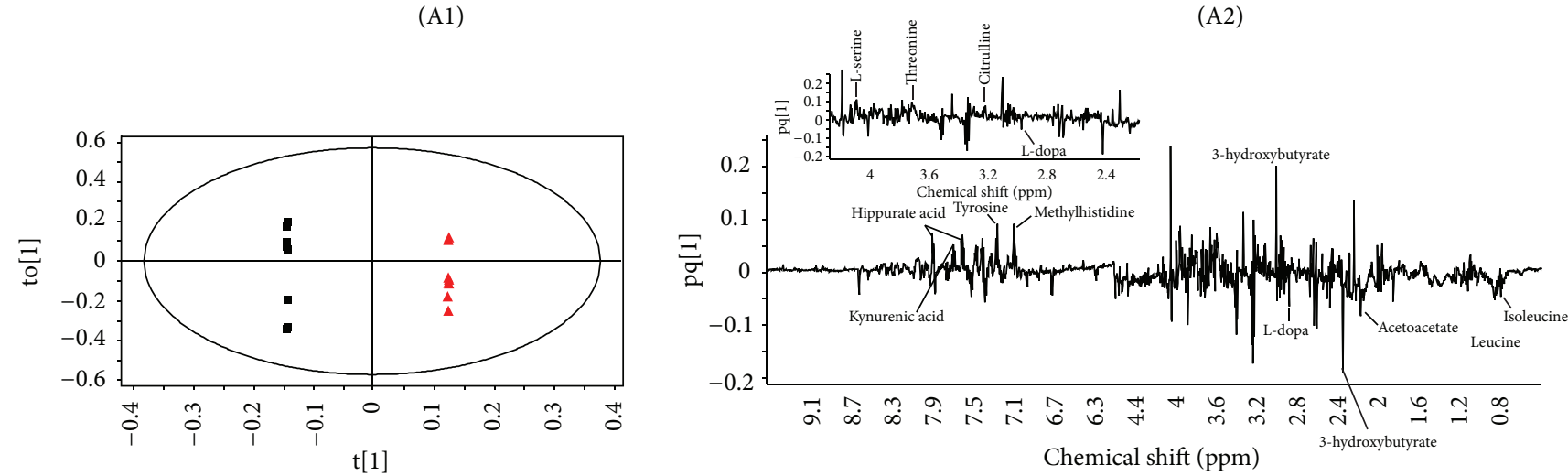

- Control

A Model

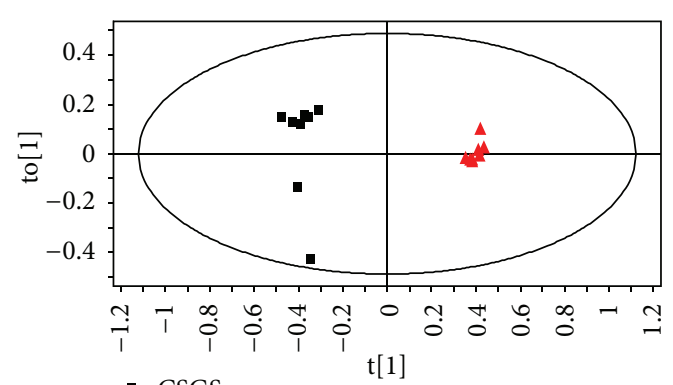

- CSGS

$\triangle$ Model
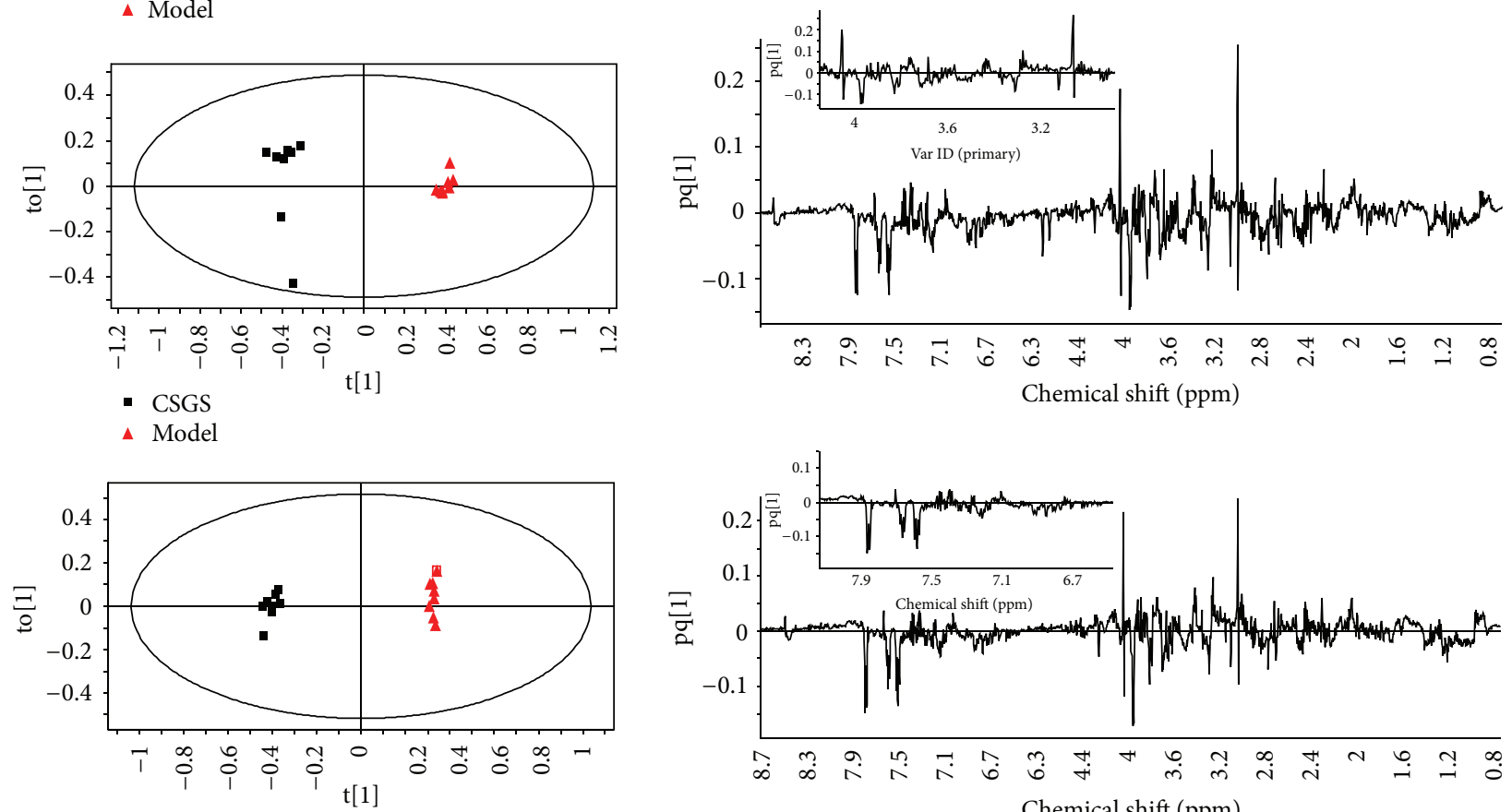

- QZ

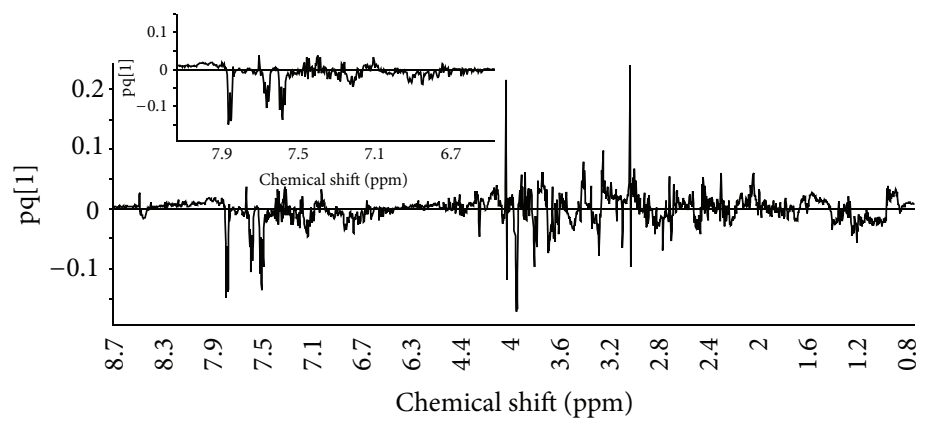

\ Model

FIGURE 2: Continued. 

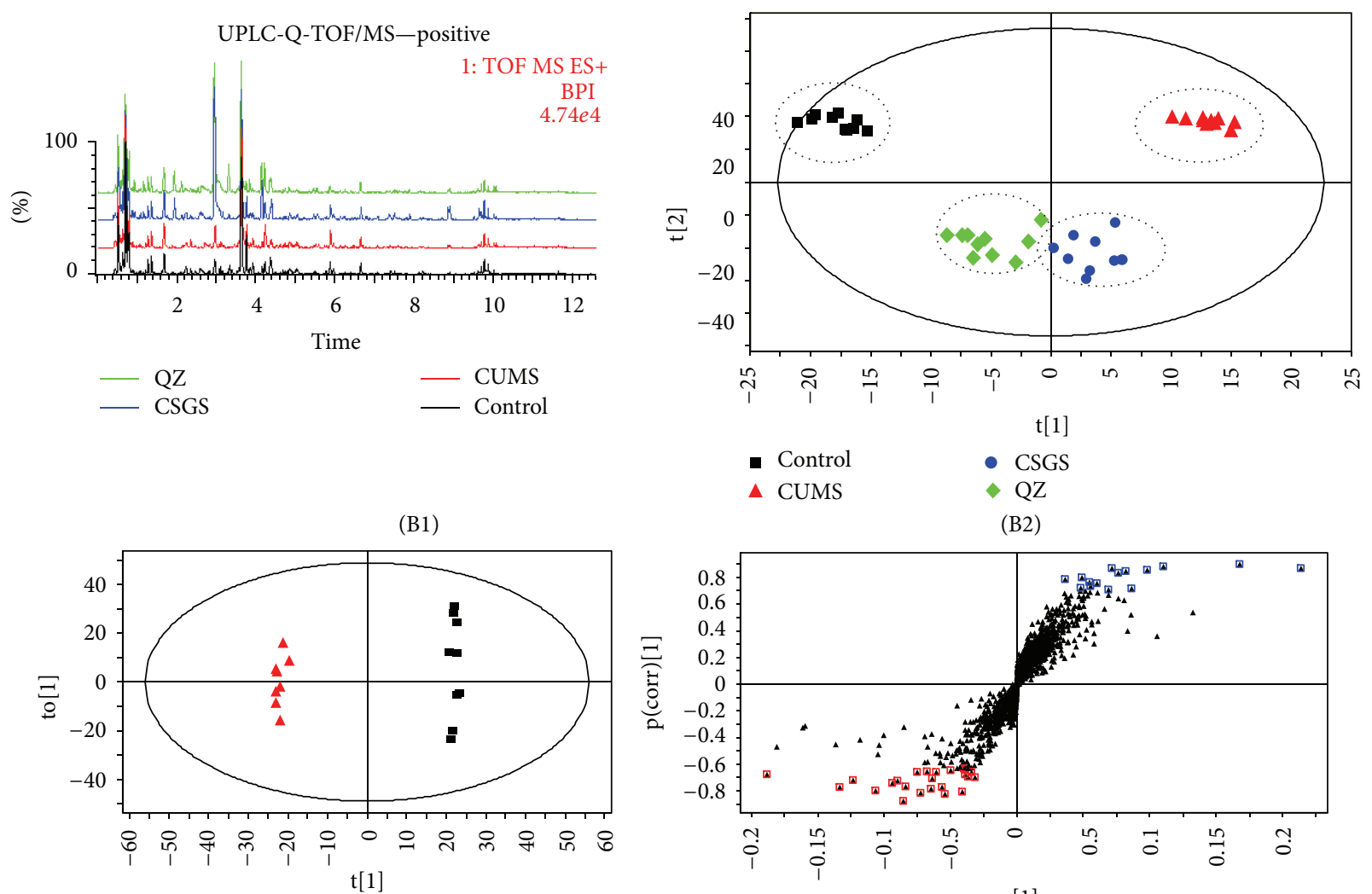

- Control

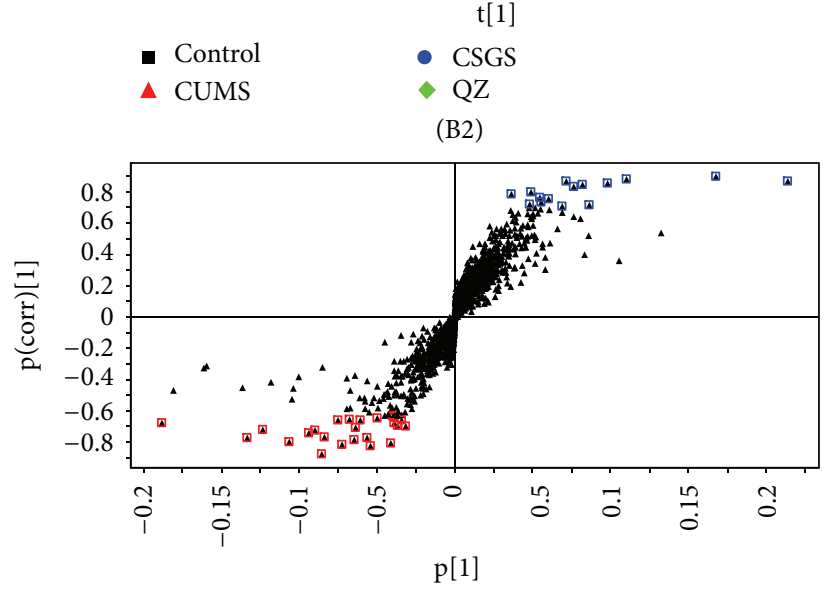

a Model
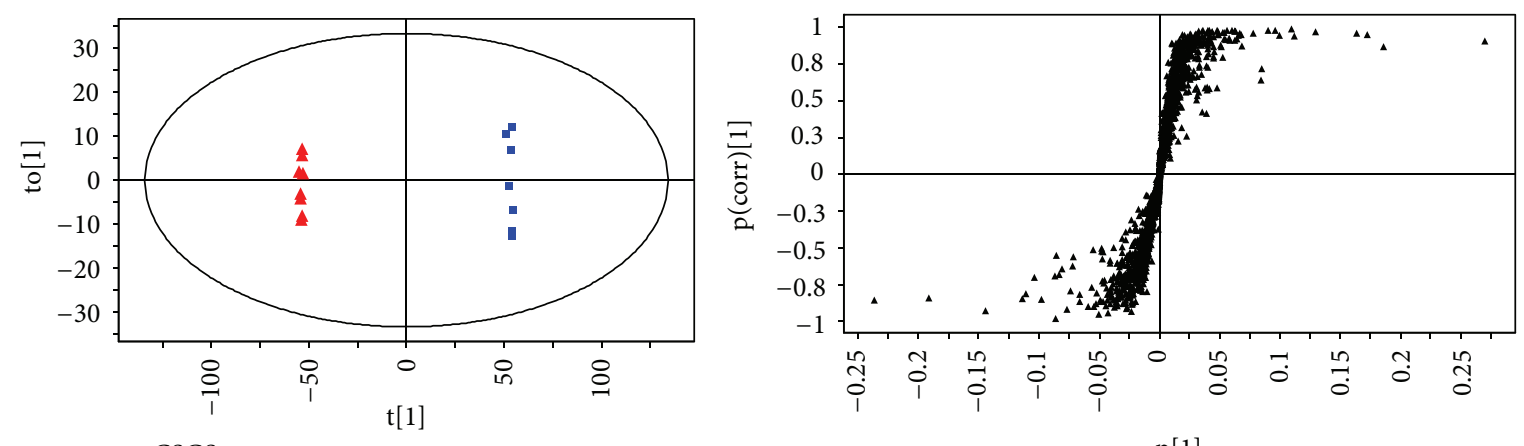

- CSGS

$\triangle$ Model

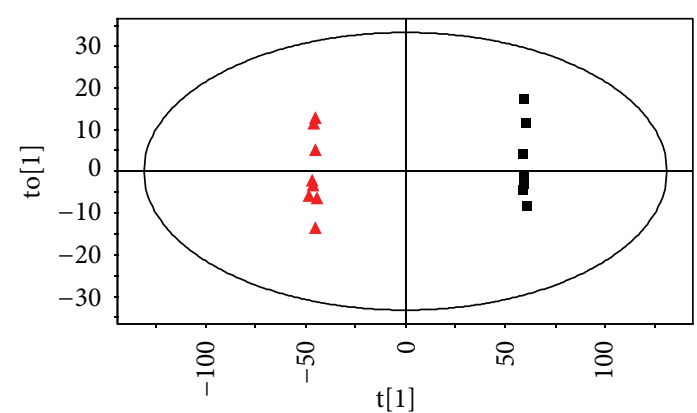

- QZ

$t[1]$

\ Model

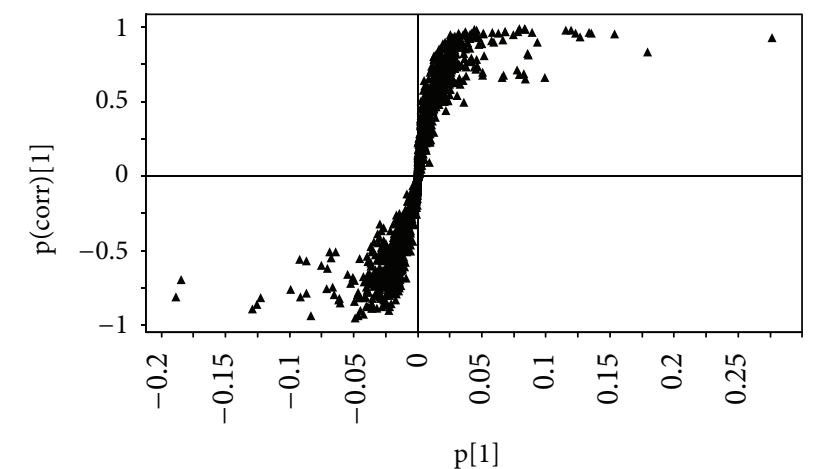

(B3)

FIgURE 2: Continued. 

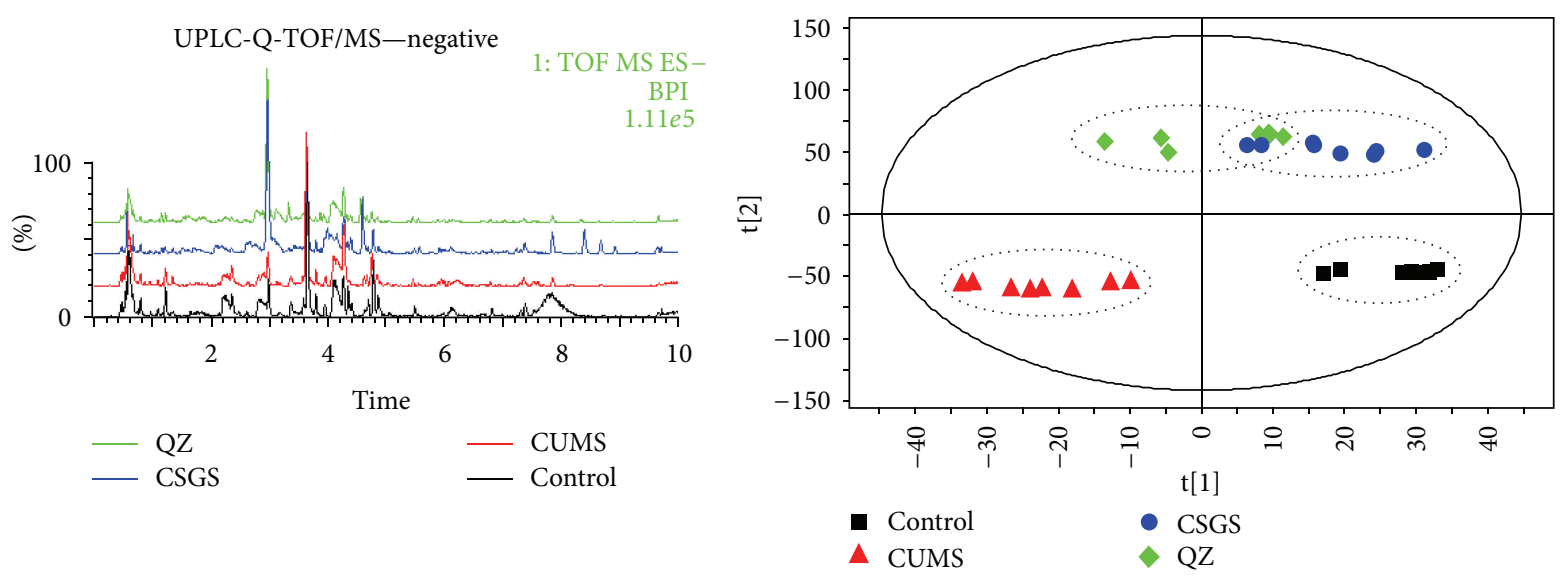

(C1)
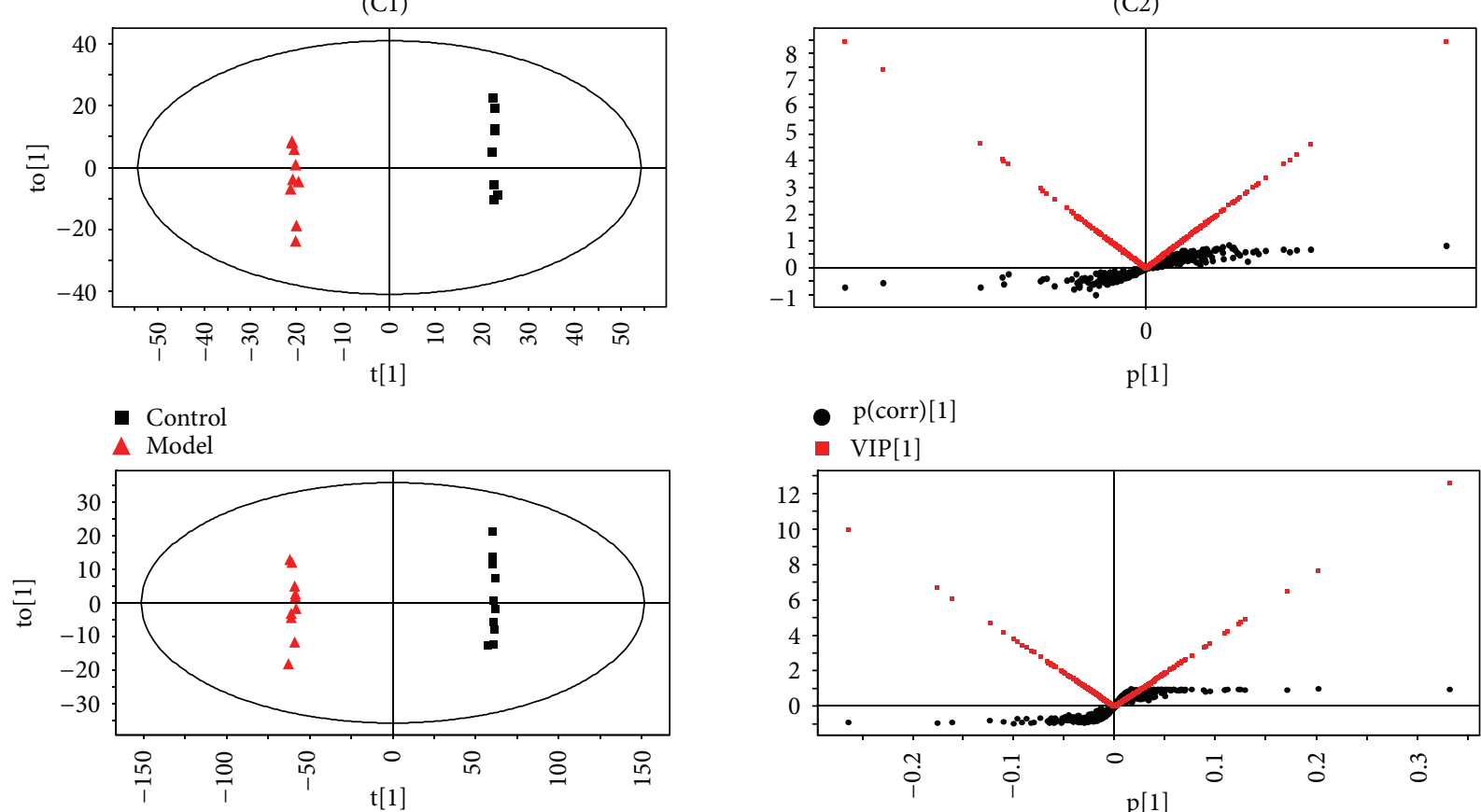

$\mathrm{p}($ corr $)[1]$

- VIP[1]
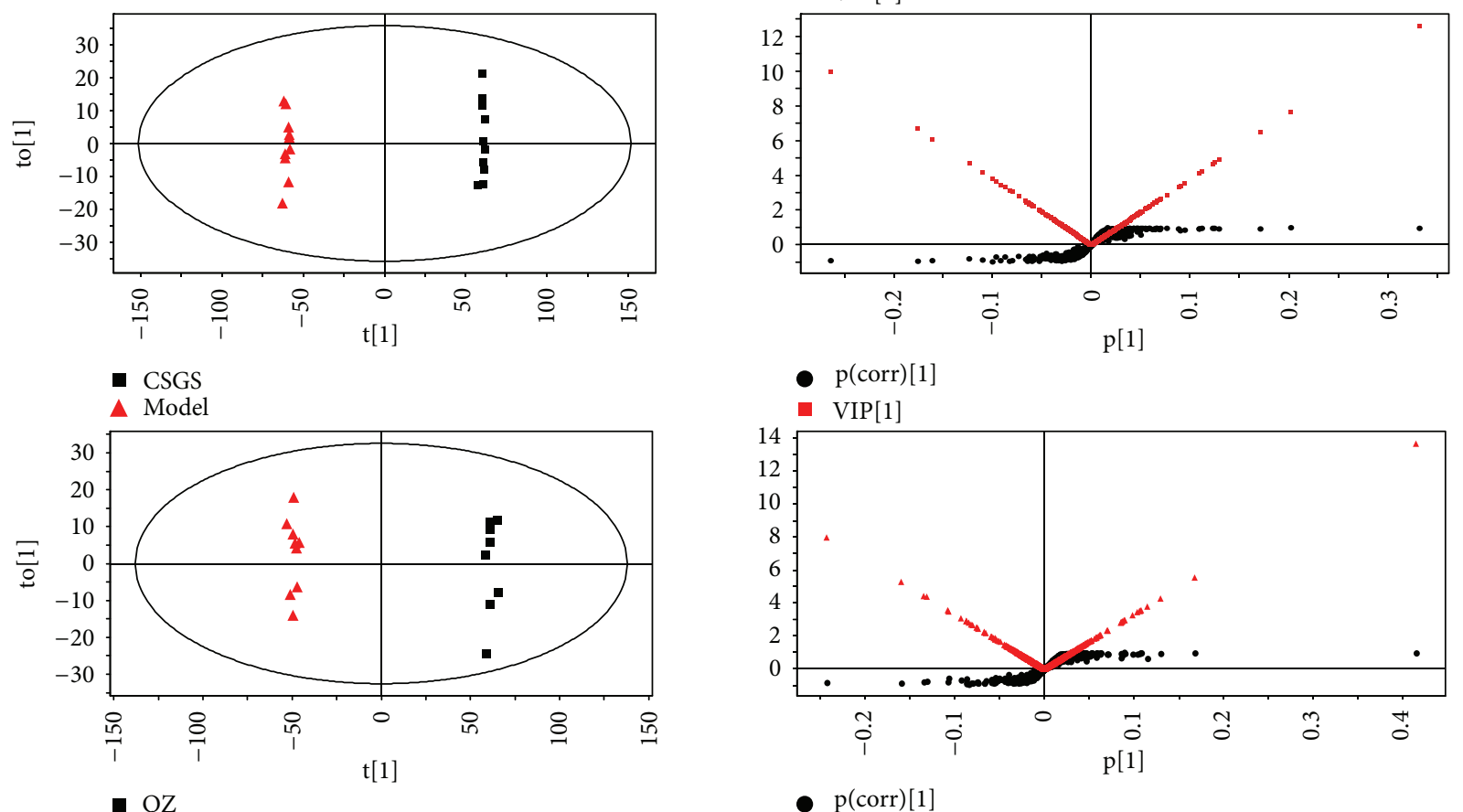

$\triangle$ Model

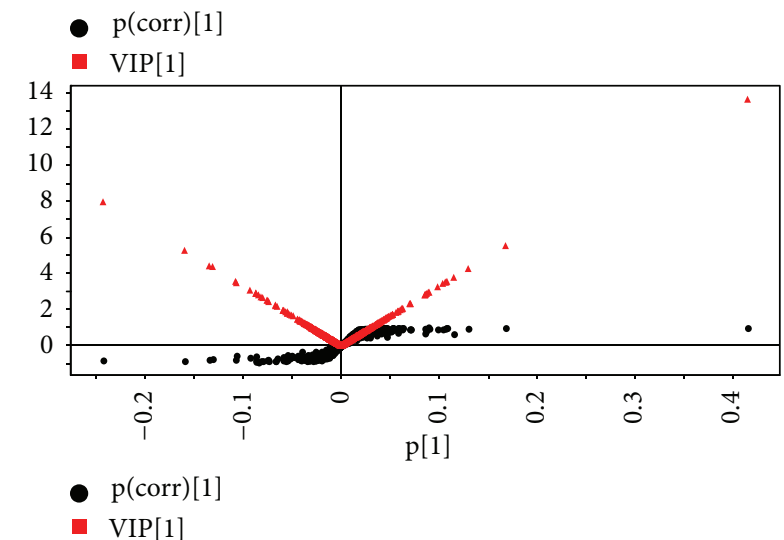

(C3)

(C4)

FIGURE 2: MVA of urine samples from all groups detected by NMR and UPLC-Q-TOF/MS. A1, B1, and C1: the spectra and chromatograms of urine samples. Black color stands for control group, red color for CUMS group, blue color for CSGS treated group, and green color for QZ treated group; A2, B2, and C2. The score plots of control, CUMS, CSGS, and QZ groups; A3, B3, and C3. OPLS-DA scores plots of CUMS versus model groups, CUMS versus CSGS groups, CUMS versus QZ groups (A3: $Q 2 X_{\mathrm{CUM}}=0.879, R^{2} Y_{\mathrm{CUM}}=0.998, Q_{(\mathrm{CUM})}^{2}=0.607, \mathrm{~B} 3$ : $\left.Q^{2} X_{(\mathrm{CUM})}=0.604, R^{2} Y_{\mathrm{CUM}}=0.994, Q_{(\mathrm{CUM})}^{2}=0.99 ; \mathrm{C} 3: Q^{2} X_{(\mathrm{CUM})}=0.659, R^{2} Y_{\mathrm{CUM}}=0.999, Q_{(\mathrm{CUM})}^{2}=0.999\right) ; \mathrm{A} 4$, B4, and C4: line plots, S-plots, and VIP-value plots of urine samples detected by NMR and UPLC-Q-TOF/MS, respectively. 


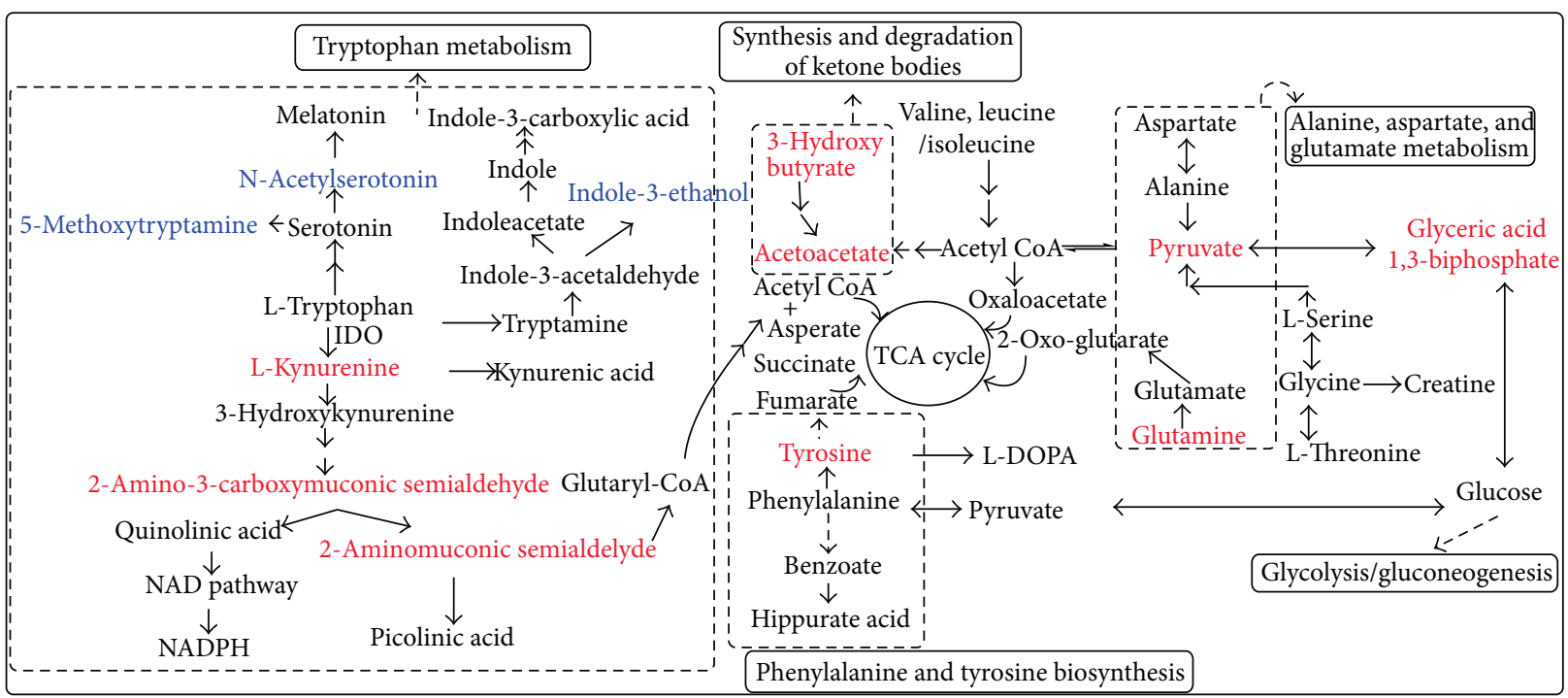

(a)
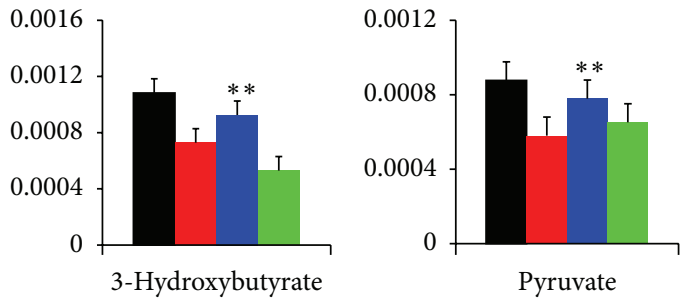

Pyruvate

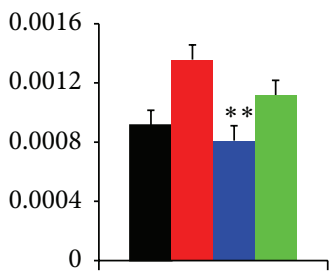

Tyrosine

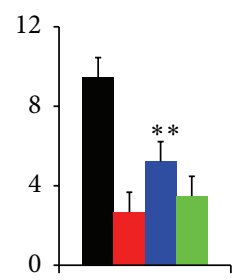

Glyceric acid 1,3-biphosphate

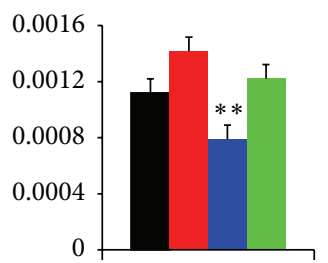

Glutamine

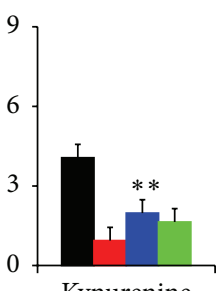

Kynurenine
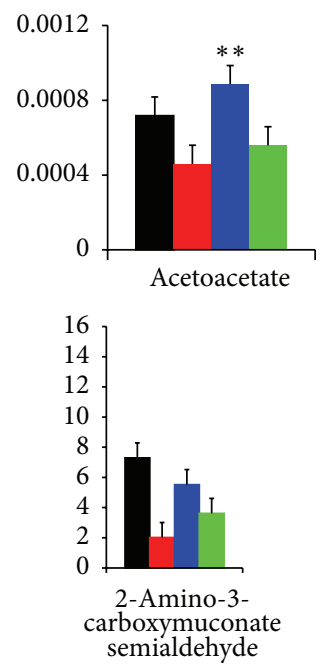
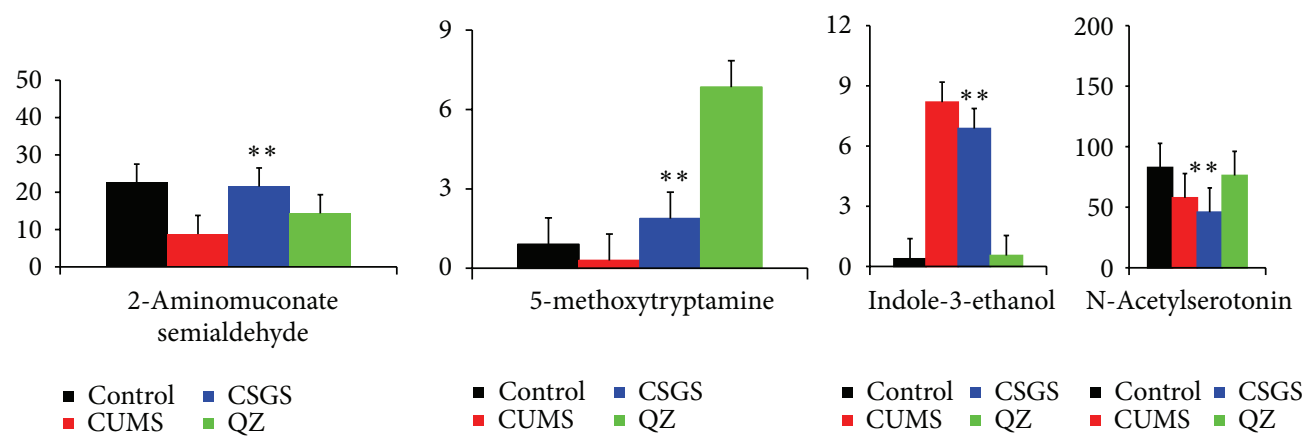

(b)

FIgURE 3: (a) Urinary metabolic pathways of CUMS-induced depression with CSGS or QZ treatment. Metabolites in red font: the potential biomarkers on which CSGS had regulations but QZ did not; metabolites in blue font: the potential biomarkers on which QZ had regulations but CSGS did not. (b) The levels of 12 potential biomarkers for differentiating CSGS and QZ treated. $\left({ }^{*} P<0.05\right.$ versus CUMS group, ${ }^{* *} P<$ 0.01 versus CUMS group). 


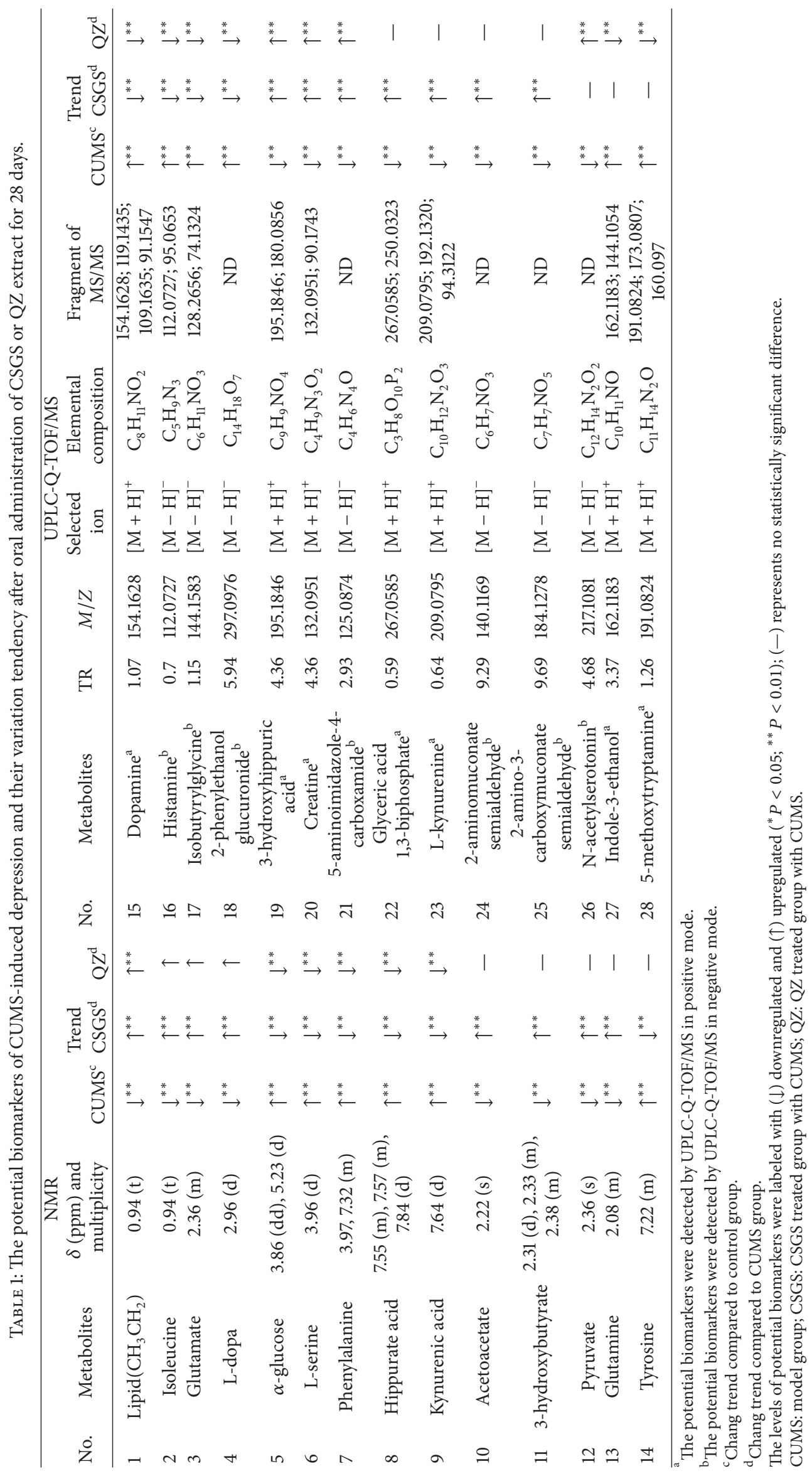


3.3. Identification of the Chemical Constitutes Responsible for Those Regulations of CSGS Missed in QZ. Further, comparison of the chemical profiles among CSGS, QZ, and Zhi-Qiao was conducted for identification of the chemical constituents responsible for those mediation effects of CSGS lost in QZ, that is, regulations on the metabolites $\mathbf{2 - 4}, \mathbf{1 0 - 1 4}$, and $22-$ 25. The extracts of CSGS, QZ (CSGS without Zhi-Qiao), and Zhi-Qiao were analyzed by using UPLC-Q-TOF/MS under the same conditions (Figures S4 and S5). Compared with the chemical profile of CSGS, some of the peaks disappeared and the intensities of some peaks were changed in QZ extracts. OPLS-DA was applied in finding the differences between CSGS and QZ extracts in the chemical profile. The variables responsible for the differentiation between the CSGS and QZ extracts were identified from S-plots; the $Y^{+}$axis represented the QZ extract; the $Y^{-}$axis represented the CSGS extract; the $X$-axis represented the number of detected ions (Figure 4(a)). Three candidate ions with retention time and $\mathrm{m} / z$ pairs at 0.57_191.0937 (i), 5.94_579.1701 (ii), and 6.94_609.1820 (iii) located furthest from the origin in the $Y^{-}$axis, contributing significantly to discriminating CSGS from QZ. In the trend plots (Figure 4(b)), the concentrations of those compounds in QZ extract were nearly zero, which indicated that these compounds came from the subtracted herb, Zhi-Qiao. Based on the retention behaviors, accurate molecular weight and MS/MS fragments from UPLC-Q-TOF/MS analysis on ZhiQiao extract (Figure 3(c)), and by comparison with reference standards, were identified as synephrine (i), naringin (ii), and hesperidin (iii-1)/neohesperidin (iii-2), respectively. They are the chemical contributors to discriminate CSGS from QZ extracts and also are the active constituents of CSGS from Zhi-Qiao to mediate the deviations of the metabolites 2-4, 10-14, and 22-25 induced by CUMS.

3.4. Active Evaluation of the Active Constituents from ZhiQiao against Monoamine Oxidase A (MAO-A). Monoamine oxidase A (MAO-A), an enzyme presenting in the outer mitochondrial membrane of neuronal and nonneuronal cells, catalyses the oxidative domination of primary, secondary, and tertiary amines [20]. The regulation of MAO-A activity appeared to play a central role in several psychiatric and neurological disorders. Thus, inhibition of MAO-A may alleviate symptoms of depression [21].

The inhibition of synephrine, naringin, hesperidin, and neohesperidin against MAO-A activity was assayed by employing rat MAO-A as enzyme source and moclobemide as positive control. Naringin showed the highest activity with an $\mathrm{IC}_{50}$ value of $5.82 \mu \mathrm{M}$ (Table 2). The $\mathrm{IC}_{50}$ values of synephrine, hesperidin, and neohesperidin were $15.23 \mu \mathrm{M}$, $26.72 \mu \mathrm{M}$, and $93.76 \mu \mathrm{M}$.

3.5. Molecular Docking Studies. Molecular docking with the MOE 2008 was carried out with the aim of understanding the possible binding orientations of naringin within the MAO active sites. The crystallographic structures of human MAOA cocrystallized with harmine (PDB entry: 2Z5X) served as protein model. The protein model and docking protocol have previously been shown to be appropriate for predicting binding orientations of ligands in the active sites of MAO-A.
TABLE 2: Inhibition of synephrine, naringin, hesperidin, and neohesperidin against MAO-A activity.

\begin{tabular}{lcc}
\hline No. & Compounds & $\mathrm{IC}_{50}(\mu \mathrm{M})$ \\
\hline i & Synephrine & 15.23 \\
ii & Naringin & 5.82 \\
iii-1 & Hesperidin & 26.72 \\
iii-2 & Neohesperidin & 93.76 \\
Control & Moclobemide & 5.25 \\
\hline
\end{tabular}

Primary experiment has indicated that naringin was poorly absorbed from the gastrointestinal tract in its original form and must be hydrolyzed by microflora enzymes (bacterial $\beta$ glucuronidase) in gut to its aglycone (naringenin) in human and rat $[22,23]$. Thus, naringenin was subjected molecular docking with the MOE 2008. We found that the aglycone of naringin was able to form a relatively stable docking structure with MAO-A through our docking experiment.

The highest ranked solution of harmine (positive control) and naringenin within the MAO-A active site was illustrated in Figures 5(a) and 5(b). The results showed that both naringenin and harmine bond within the substrate cavity of the enzyme through heteroatom of the heterocyclic and water molecule as a bridge which linked activity compounds and enzyme. As shown in Figures 5(b) and 5(c), the benzene ring of the naringenin binds within the substrate cavity of the enzyme and undergoes potential hydrogen bonding with FAD cofactor by a water molecule. In addition, the oxygen atom of pynan ring in naringenin undergoes potential hydrogen bonding with the phenolic hydrogen of Ile-207, Asn-181 which was shown in Figure 5(b). Furthermore, the phenolic hydroxyl of benzene ring of naringenin undergoes potential hydrogen bonding with the phenolic hydrogen of Phe-208, Ala-111. The experimental results showed that naringenin is able to connect steadily with MAO-A through water molecule and could be viewed as a promising compound for the inhibition of MAO-A activity.

\section{Discussion}

Traditional Chinese herbal medicine typically uses mixtures of many herbs to treat a given disease. It is believed that complex interactions produce synergistic effects and reduce possible side effects from some herbs. The conventional strategy applied in screening bioactive components in TCMs is isolation and identification of the individual components one by one and then evaluation of their activities in vitro/in vivo. This strategy is not only time consuming and labor intensive, but also often fails to discover the bioactive constituents contributing to the efficacy of TCM formulas. A very important reason is that no synergistic effects among diverse chemical constituents in herbal medicines are considered.

Chaihu-Shu-Gan-San (CSGS) has been proved to be an effective TCMs formula for treatment of depression and is featured as multiingredients preparation and multitargets intervention on the systemic level [11]. However, the chemical constituents of CSGS related to its efficacy were still not clear, even though some constituents with antidepressant 
S-plot $(\mathrm{CSGS}=-1, \mathrm{QZ}=1)$

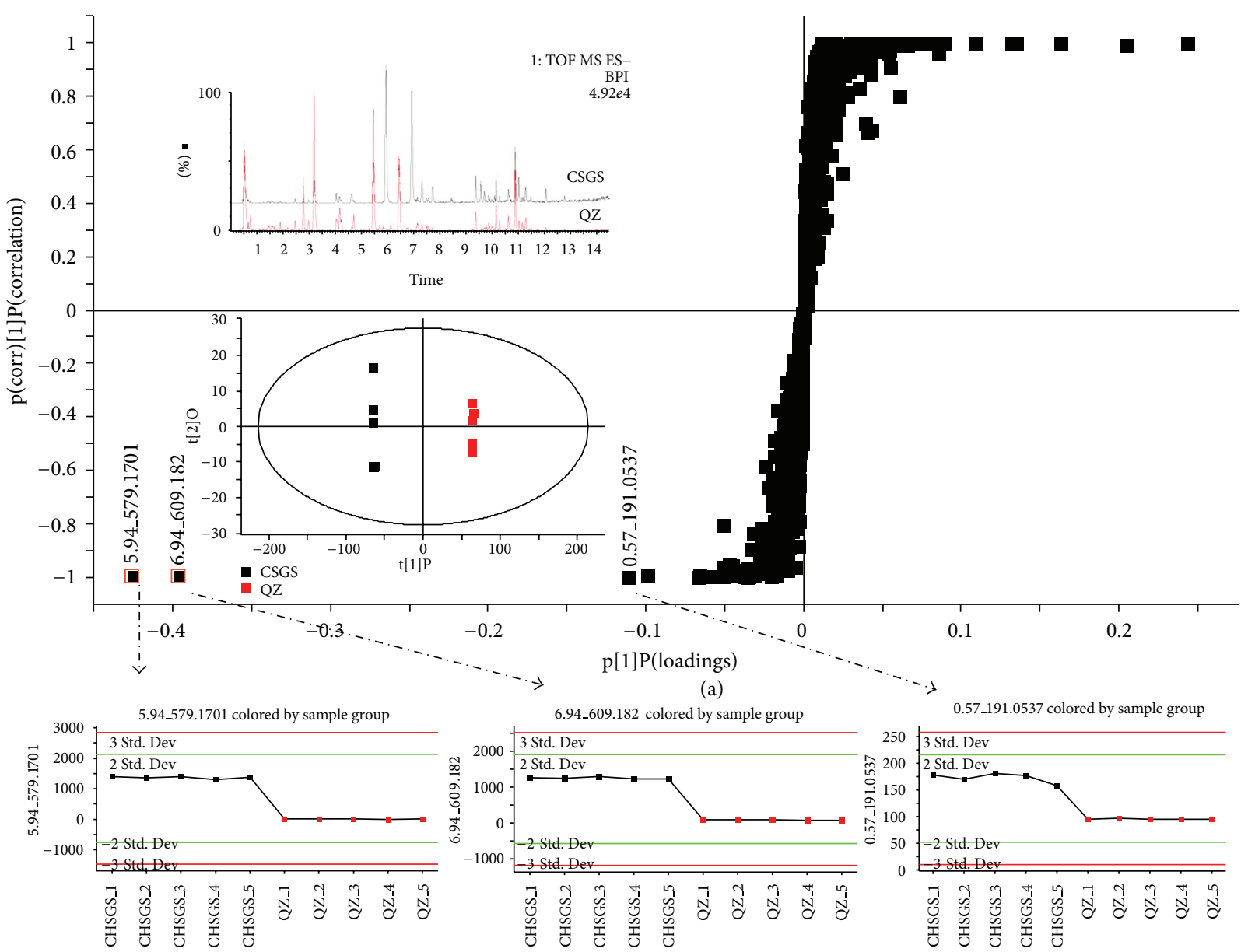

(b)
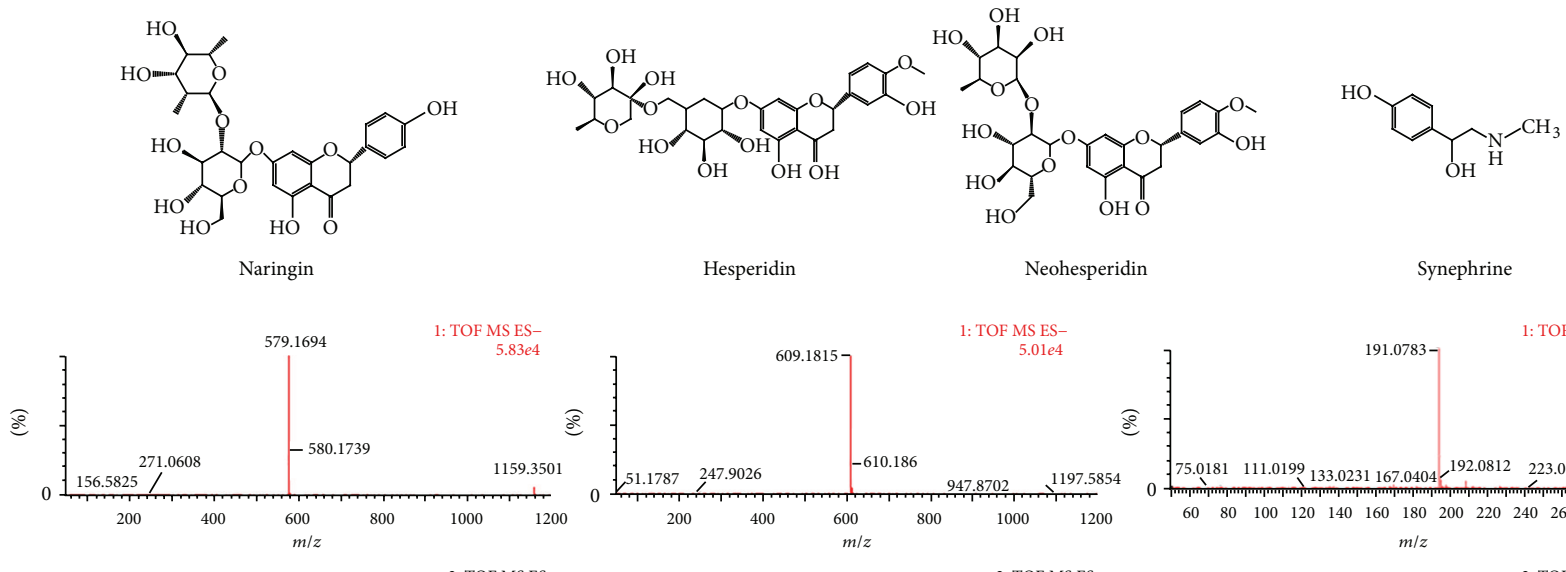

idin Synephrine
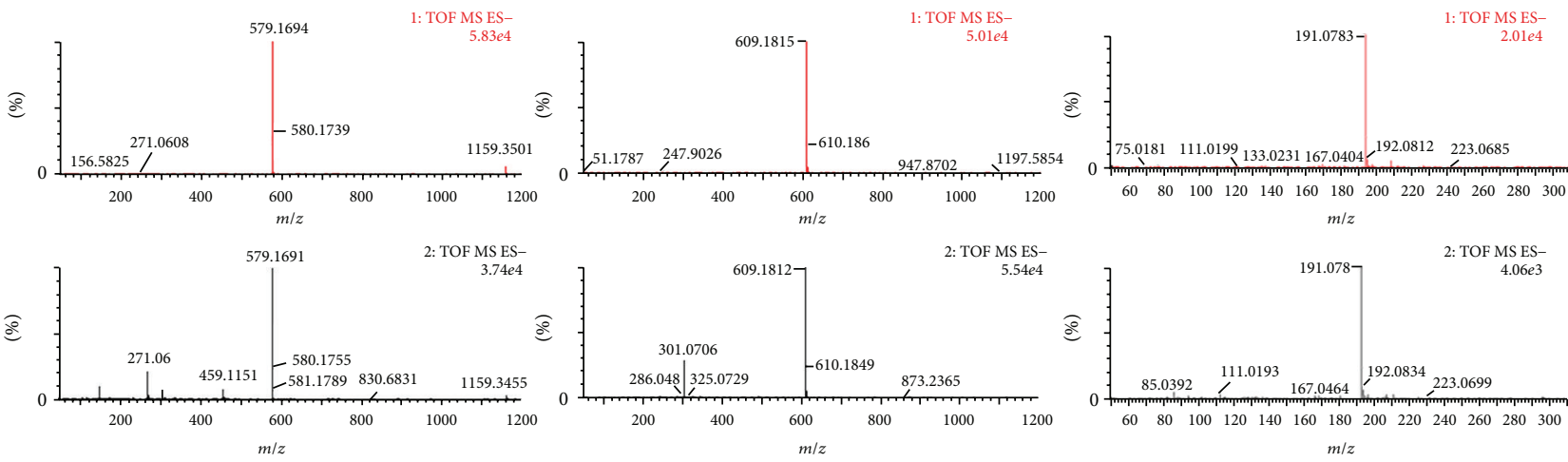

$\mathrm{m} / \mathrm{z}$

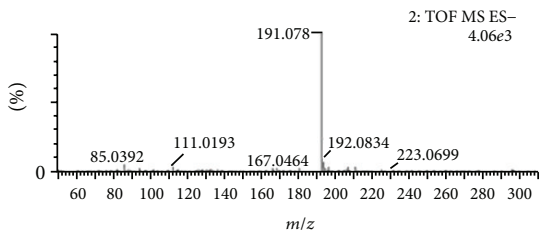

(c)

FIGURE 4: MVA on chemical profiles of the CSGS and QZ extracts. (a) Score plots in OPLS-DA analysis of CSGS and QZ. (b) Trend plots: the level of the variables in the CSGS (black spot) and QZ extracts (red spot). (c) The structures and MS spectra of synephrine, naringin, and hesperidin/neohesperidin. 


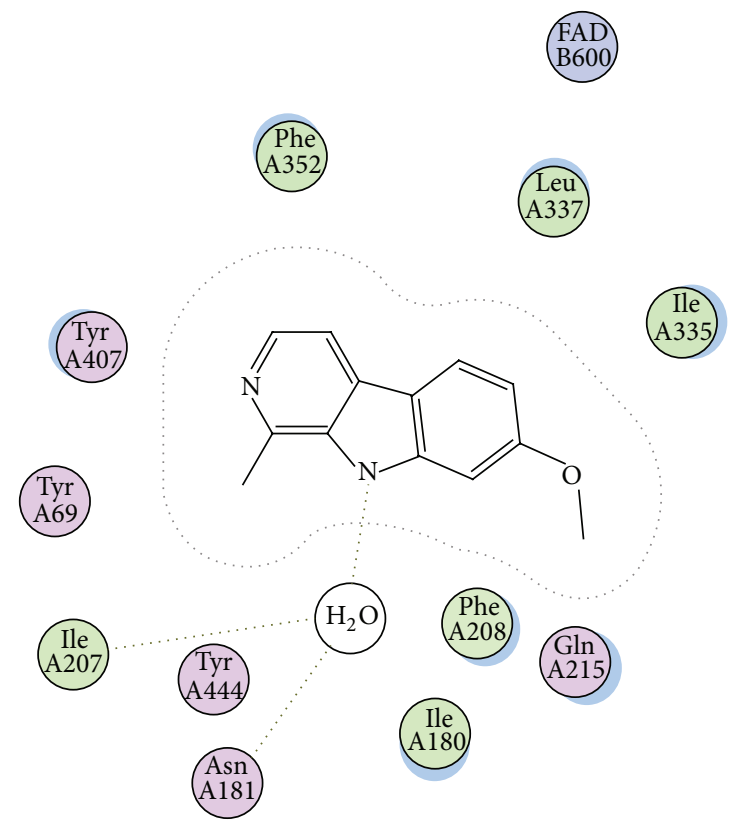

(a)

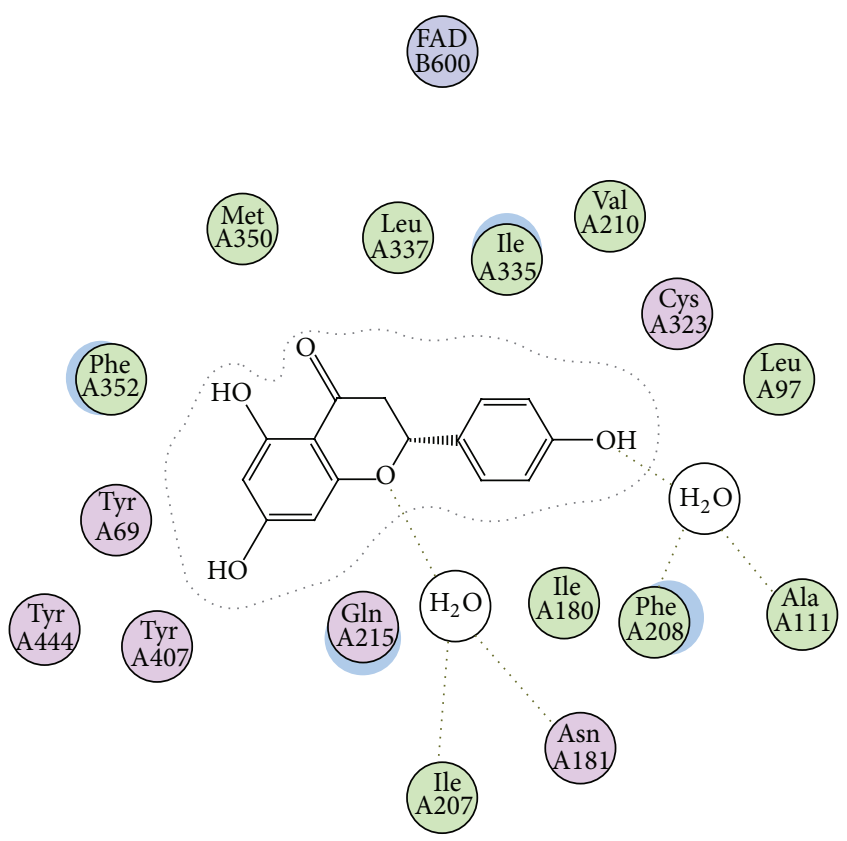

(b)

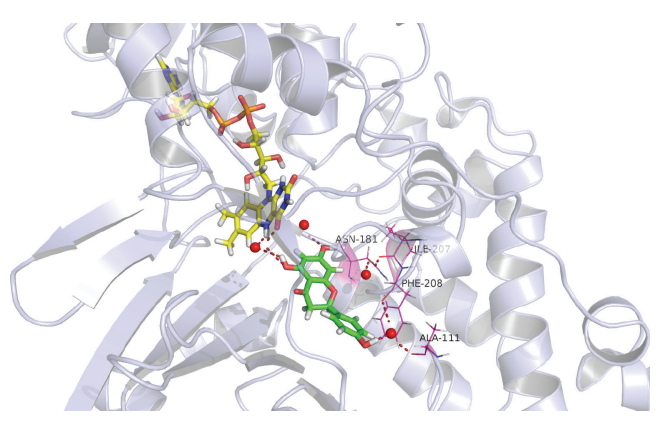

(c)

FIgURE 5: Computer-aided molecular docking. (a) The 2D map of predicted binding orientation of harmine within the MAO-A active site. (b) The 2D map of predicted binding orientation of naringenin within the MAO-A active site. (c) The 3D map of predicted binding mode of naringenin within the active site of MAO-A.

effect were reported from the single herbs of CSGS, such as saikosides (Chai-Hu) [24], total glycosides of paeonia (TGP, Bai-Shao) [25], and liquiritin (Gan-Cao) [26].

Metabonomics is focused on measuring the overall metabolites of biological samples which coincides with the holistic thinking of TCM. It enables the parallel assessment of the levels of a broad range of endogenous and exogenous metabolites [27] and has shown potential in evaluation of therapeutic effect of TCMs [9].

In our research, metabonomics provided a visualized pattern and relative quantitative estimate to help us acknowledge the potential biomarkers of CUMS induced depression and the different regulations after CSGS and QZ treatments. The results-indicated that CSGS showed more comprehensive regulations than QZ after 28 days of therapeutic intervention. QZ did not show effect on twelve potential biomarkers (2-4, 10-14, 22-25) as CSGS did. The different regulations between CSGS and QZ treated groups suggested that the subtracted herb, that is, Zhi-Qiao, was responsible for the missing regulations of QZ compared to CSGS. The findings indicated that Zhi-Qiao contributed to the antidepressant effect of CSGS through regulating the deviations of the potential biomarkers 2-4, 10-14, 22-25 (Table 1 and Figure 3). Among them, isoleucine (2), glutamate (3), dopa (4), acetoacetate (10), 3-hydroxybutyrate (11), pyruvate (12), glutamine (13), tyrosine (14), and glyceric acid 1, 3-biphosphate (22) were involved in metabolic pathways associated with the onset of CUMS-induced depression in our previous study [10], including ketone bodies synthesis, phenylalanine, tyrosine and tryptophan biosynthesis, valine, aspartate and glutamate metabolism, and glycolysis/gluconeogenesis. The other three potential biomarkers, kynurenine (23), 2-aminomuconate semialdehyde (24), and 2-amino-3-carboxymuconate semialdehyde (25), are the main products in kynurenine pathway (a major route of tryptophan metabolism). Tryptophan and its metabolites such as serotonin and kynurenines (KYNs) are strong modulators of emotional behavior in the central nervous system (CNS) [28] and have been implicated in 
brain dysfunction in several disorders such as Huntington's disease, Alzheimer's disease, and depression [28-30]. Obviously, Zhi-Qiao participated in the regulation of tryptophan metabolism, ketone bodies synthesis, phenylalanine, tyrosine and tryptophan biosynthesis, valine, aspartate, glutamate metabolism and glycolysis/gluconeogenesis to assist CSGS treated of depression.

Interestingly, QZ showed regulations on three of the potential biomarkers, N-acetylserotonin (26), indole-3ethanol (27), and 5-methoxytryptamine (28) (Figure 3(b)), but CSGS did not have effects on these three metabolites. They are the products of 5-HT synthesis, one of the pathways in tryptophan metabolism. The results suggested that QZ influenced the synthesis of 5-HT because there exist active constituents in QZ (i.e., all single herbs of CSGS except ZhiQiao) and competing constituents in Zhi-Qiao. CSGS did not have effect on the synthesis of 5-HT; however, it could mediate the disturbances of tryptophan metabolism induced by CUMS through kynurenine pathways (KYP), the other pathways in tryptophan metabolism.

Undoubtedly, the mediations of CSGS missed in QZ should come from Zhi-Qiao, the single herb subtracted from CSGS. However, not all constituents in Zhi-Qiao make contributions to those missing medications. In order to know which compounds from Zhi-Qiao are associated with those missing medications, MVA was performed on the chemical profiles of CSGS and QZ extracts. Four variables shown in the S-plots of OPLS-DA were recognized as significant contributors to discriminate the chemical profiles of CSGS and QZ extracts. They represented the antidepressant ingredients of CSGS from Zhi-Qiao, and were identified as synephrine, naringin, hesperidin, and neohesperidin, respectively.

To the best of our knowledge, this is the first report for the antidepressant activity naringin, hesperidin, and neohesperidin. Antidepressant-like effect of synephrine was tested in mouse models of immobility tests [31]. The in vitro MAOA inhibitory assay confirmed their antidepressant effect using rat MAO-A as enzyme source and moclobemide as positive control. Naringin showed the highest activity with an $\mathrm{IC}_{50}$ value of $5.82 \mu \mathrm{M}$, while synephrine, hesperidin, and neohesperidin were found to be relatively weak. The possible binding orientation of naringin within the MAO active sites was speculated by molecular docking study and the results suggested that the aglycone of naringin and naringenin [21, 22] was fused with MAO-A by a hydrogen bond through water molecule.

\section{Conclusions}

This paper integrated metabonomics and chemical profile in combination with the multivariate statistical analysis (MVA) to discover the chemical constituents which contribute to the efficacy of CSGS. Through the difference between CSGS and QZ extracts in chemical profiles and the regulations of metabolic perturbations induced by CUMS, four compounds were identified as the active constituents of CSGS contributed by Zhi-Qiao. They were responsible for those regulations of CSGS on the deviations of the metabolites 2-4, 10-14, and 22-25, involved in metabolic dysfunction of ketone bodies synthesis, phenylalanine, tyrosine and tryptophan biosynthesis, valine, aspartate, glutamate metabolism, and glycolysis/gluconeogenesis. The potential antidepressant activities of the four compounds were evaluated by MAO-A activity assay in vitro. Naringin showed the potent inhibition against $\mathrm{MAO}-$ A activity with an $\mathrm{IC}_{50}$ value of $5.82 \mu \mathrm{M}$.

Accordingly, we ascertained the contributions of ZhiQiao to the CSGS antidepressant effect. In future, the integration of metabonomics and chemical profile could be used to clarify the contributions of all single herbs in CSGS and uncover all active constituents of CSGS. Our findings provide a new effective strategy for uncovering what the active chemical constituents in TCM formula are and how they make contributions for the efficacy of the formula.

$\begin{array}{ll}\text { Abbreviations } \\ \text { CSGS: } & \text { Chaihu-Shu-Gan-San } \\ \text { MVA: } & \text { Multivariate statistical analysis } \\ \text { Zhi-Qiao: } & \text { Citrus aurantium L. } \\ \text { QZ: } & \text { Chaihu-Shu-Gan-San without Zhi-Qiao } \\ \text { CUMS: } & \text { Chronic unpredicted mild stress } \\ \text { MAO-A: } & \text { Monoamine oxidase A } \\ \text { TCMs: } & \text { Traditional Chinese medicines } \\ \text { QC: } & \text { Quality control } \\ \text { TSP: } & \text { Sodium-3-trimethylsilyl-[2, 2, 3, } \\ & \left.\text { 3- }{ }^{2} \mathrm{H}_{4}\right]-1-\text { propionate } \\ \text { PCA: } & \text { Principal components analysis } \\ \text { OPLS-DA: } & \text { Orthogonal to partial least } \\ & \text { squares-discriminate analysis } \\ \text { CNS: } & \text { Central nervous system } \\ \text { RT: } & \text { Retention time. }\end{array}$

\section{Conflict of Interests}

The authors declare no conflict of interests.

\section{Acknowledgment}

This work was financially supported by the National Natural Science Foundation of China (81173512) and the National S \& T Major Special Project on Major New Drug Innovation (2013ZX09508104 and 2012ZX09301002-001). They gratefully acknowledge the PyMol software team and Chinese Academy of Medical Sciences Ultraperformance Computing Center for providing the software of MOE 2008.

\section{References}

[1] D. Normile, "The new face of traditional Chinese medicine," Science, vol. 299, no. 5604, pp. 188-190, 2003.

[2] J. L. Griffin, L. A. Walker, S. Garrod, E. Holmes, R. F. Shore, and J. K. Nicholson, "NMR spectroscopy based metabonomic studies on the comparative biochemistry of the kidney and urine of the bank vole (Clethrionomys glareolus), wood mouse (Apodemus sylvaticus), white toothed shrew (Crocidura suaveolens) and the laboratory rat," Comparative Biochemistry and Physiology B, vol. 127, no. 3, pp. 357-367, 2000. 
[3] A. H. Liu, Y. H. Lin, M. Yang et al., "Development of the fingerprints for the quality of the roots of Salvia miltiorrhiza and its related preparations by HPLC-DAD and LC-MSn," Journal of Chromatography B, vol. 846, no. 1-2, pp. 32-41, 2007.

[4] J. Jing, C. O. Chan, L. Xu et al., "Development of an inline HPLC fingerprint ion-trap mass spectrometric method for identification and quality control of Radix Scrophulariae," Journal of Pharmaceutical and Biomedical Analysis, vol. 56, no. 4, pp. 830-835, 2011.

[5] F. Dieterle, B. Riefke, G. Schlotterbeck et al., "NMR and MS methods for metabonomics," Methods in Molecular Biology, vol. 691, pp. 385-415, 2011.

[6] M. Coen, E. Holmes, J. C. Lindon, and J. K. Nicholson, "NMR-based metabolic profiling and metabonomic approaches to problems in molecular toxicology," Chemical Research in Toxicology, vol. 21, no. 1, pp. 9-27, 2008.

[7] Y. Dai, Z. Li, L. Xue et al., "Metabolomics study on the anti-depression effect of xiaoyaosan on rat model of chronic unpredictable mild stress," Journal of Ethnopharmacology, vol. 128, no. 2, pp. 482-489, 2010.

[8] A. Zhang, H. Sun, Z. Wang, W. Sun, P. Wang, and X. Wang, "Metabolomics: towards understanding traditional Chinese medicine," Planta Medica, vol. 76, no. 17, pp. 2026-2035, 2010.

[9] M. Wang, R. J. Lamers, H. A. Korthout et al., "Metabolomics in the context of systems biology: bridging traditional Chinese medicine and molecular pharmacology," Phytotherapy Research, vol. 19, pp. 173-182, 2005.

[10] H. W. Zhang and Z. M. Zou, "Application in clinic and research advances of ChaiHu-Shu-Gan-San," Lishizhen Medicine and Materia Medica Research, vol. 18, no. 3, pp. 1234-1236, 2007.

[11] Z. H. Su, S. Q. Li, G. A. Zou et al., "Urinary metabonomics study of anti-depressive effect of Chaihu-Shu-Gan-San on an experimental model of depression induced by chronic variable stress in rats," Journal of Pharmaceutical and Biomedical Analysis, vol. 55, no. 3, pp. 533-539, 2011.

[12] Z. H. Su, G. A. Zou, A. Preiss, H. W. Zhang, and Z. M. Zou, "Online identification of the antioxidant constituents of traditional Chinese medicine formula Chaihu-Shu-GanSan by LC-LTQ-Orbitrap mass spectrometry and microplate spectrophotometer," Journal of Pharmaceutical and Biomedical Analysis, vol. 53, no. 3, pp. 454-461, 2010.

[13] N. Gómez, D. Balsa, and M. Unzeta, "A comparative study of some kinetic and molecular properties of microsomal and mitochondrial monoamine oxidase," Biochemical Pharmacology, vol. 37, no. 18, pp. 3407-3413, 1988.

[14] W. L Delano, The PyMOL Molecular Graphics System, DeLano Scientific, SanCarlos, Calif, USA, 2002.

[15] M. P. Hodson, G. J. Dear, A. D. Roberts et al., "A gender-specific discriminator in Sprague-Dawley rat urine: the deployment of a metabolic profiling strategy for biomarker discovery and identification," Analytical Biochemistry, vol. 362, no. 2, pp. 182192, 2007.

[16] S. Bijlsma, I. Bobeldijk, E. R. Verheij et al., "Large-scale human metabolomics studies: a strategy for data (pre-) processing and validation," Analytical Chemistry, vol. 78, pp. 567-574, 2006.

[17] M. Papp, P. Willner, and R. Muscat, "An animal model of anhedonia: attenuation of sucrose consumption and place preference conditioning by chronic unpredictable mild stress," Psychopharmacology, vol. 104, no. 2, pp. 255-259, 1991.

[18] P. Willner, A. Towell, D. Sampson, S. Sophokleous, and R. Muscat, "Reduction of sucrose preference by chronic unpredictable mild stress, and its restoration by a tricyclic antidepressant," Psychopharmacology, vol. 93, no. 3, pp. 358-364, 1987.

[19] X. Zheng, A. Kang, C. Dai et al., "Quantitative analysis of neurochemical panel in rat brain and plasma by liquid chromatography-tandem mass spectrometry," Analytical Chemistry, vol. 84, pp. 10044-10051, 2012.

[20] J. C. Shih, K. Chen, and M. J. Ridd, "Monoamine oxidase: from genes to behavior," Annual Review of Neuroscience, vol. 22, pp. 197-217, 1999.

[21] J. H. Meyer, N. Ginovart, A. Boovariwala et al., "Elevated monoamine oxidase A levels in the brain: an explanation for the monoamine imbalance of major depression," Archives of General Psychiatry, vol. 63, no. 11, pp. 1209-1216, 2006.

[22] V. D. Bokkenheuser, C. H. L. Shackleton, and J. Winter, "Hydrolysis of dietary flavonoid glycosides by strains of intestinal Bacteroides from humans," Biochemical Journal, vol. 248, no. 3, pp. 953-956, 1987.

[23] C. Li, C. Yang, X. Peng, Z. Xiong, and F. Li, "Simultaneous determination of neoeriocitrin and naringin in rat plasma after oral administration of a Chinese compound formulation by UPLC-MS-MS," Journal of Chromatographic Science, vol. 48, no. 5, pp. 342-347, 2010.

[24] B. Lee, H. Y. Yun, I. Shim et al., "Bupleurum falcatum prevents depression and anxiety-like behaviors in rats exposed to repeated restraint stress," Journal of Industrial Microbiology and Biotechnology, vol. 22, no. 3, pp. 422-430, 2012.

[25] Q. Q. Mao, S. P. Ip, Y. F. Xian et al., "Peony glycosides reverse the effects of corticosterone on behavior and brain BDNF expression in rats," Behavioural Brain Research, vol. 227, no. 1, pp. 305-309, 2012.

[26] Z. Zhao, W. Wang, H. Guo, and D. Zhou, "Antidepressantlike effect of liquiritin from Glycyrrhiza uralensis in chronic variable stress induced depression model rats," Behavioural Brain Research, vol. 194, no. 1, pp. 108-113, 2008.

[27] J. K. Nicholson and J. C. Lindon, "Systems biology: metabonomics," Nature, vol. 455, no. 7216, pp. 1054-1056, 2008.

[28] G. F. Oxenkrug, "Tryptophan-kynurenine metabolism as a common mediator of genetic and environmental impacts in major depressive disorder: the serotonin hypothesis revisited 40 years later," Israel Journal of Psychiatry and Related Sciences, vol. 47, no. 1, pp. 56-63, 2010.

[29] T. W. Stone, "Kynurenines in the CNS: from endogenous obscurity to therapeutic importance," Progress in Neuro-Biology, vol. 64, pp. 185-218, 2001.

[30] T. W. Stone and L. G. Darlington, "Endogenous kynurenines as targets for drug discovery and development," Nature Reviews Drug Discovery, vol. 1, no. 8, pp. 609-620, 2002.

[31] D. K. Song, H. W. Suh, J. S. Jung, M. B. Wie, K. H. Son, and Y. H. Kim, "Antidepressant-like effects of p-synephrine in mouse models of immobility tests," Neuroscience Letters, vol. 214, no. 2-3, pp. 107-110, 1996. 


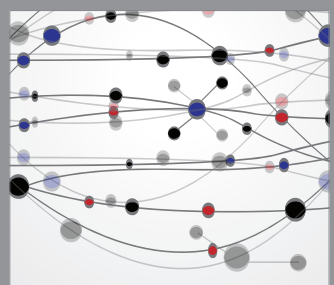

The Scientific World Journal
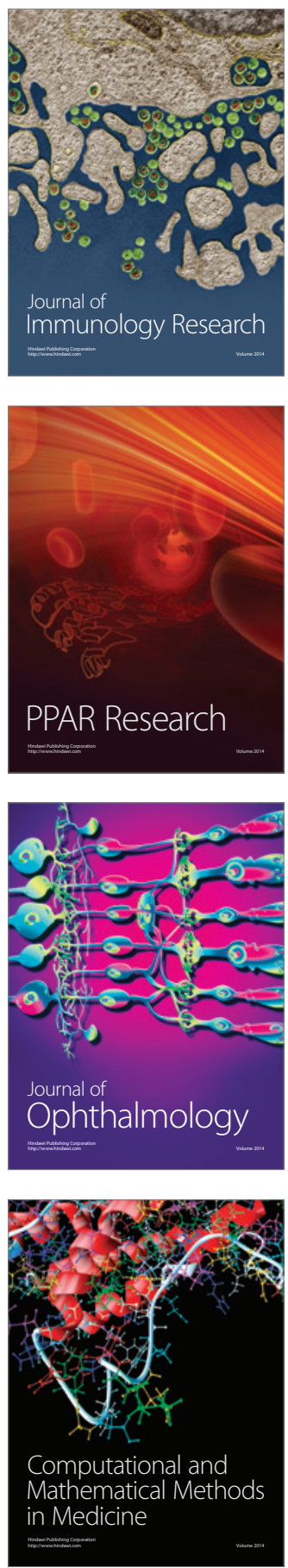

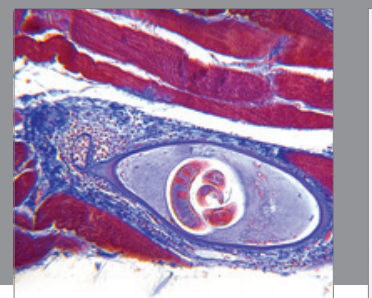

Gastroenterology

Research and Practice
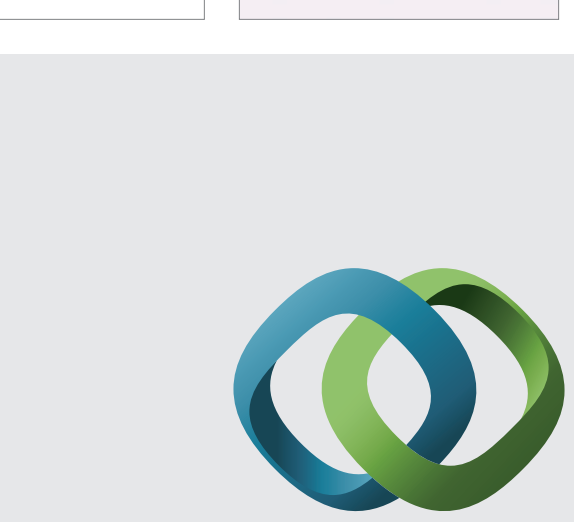

\section{Hindawi}

Submit your manuscripts at

http://www.hindawi.com
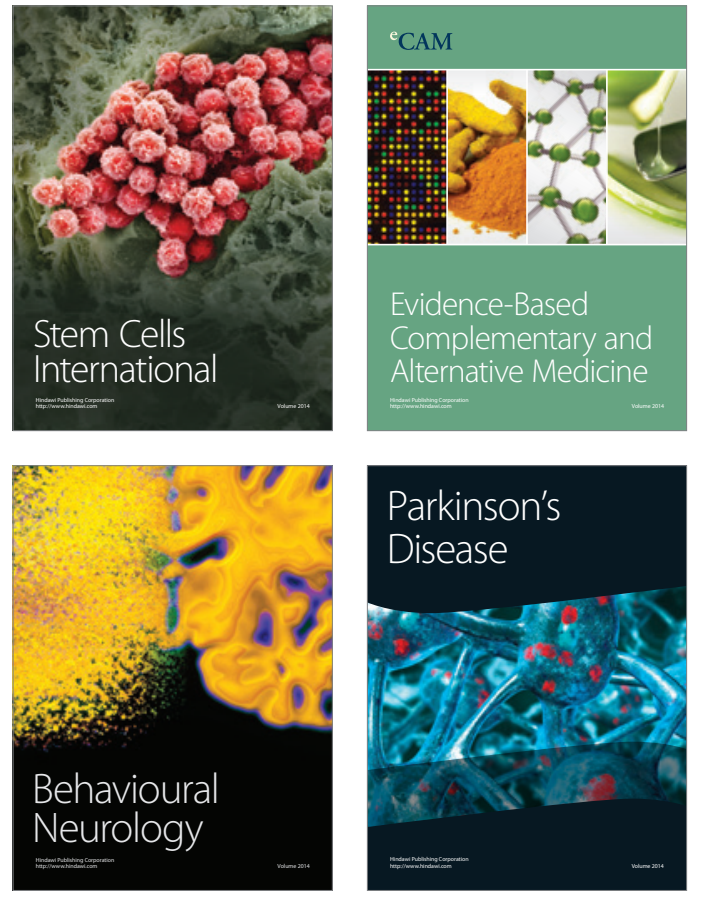
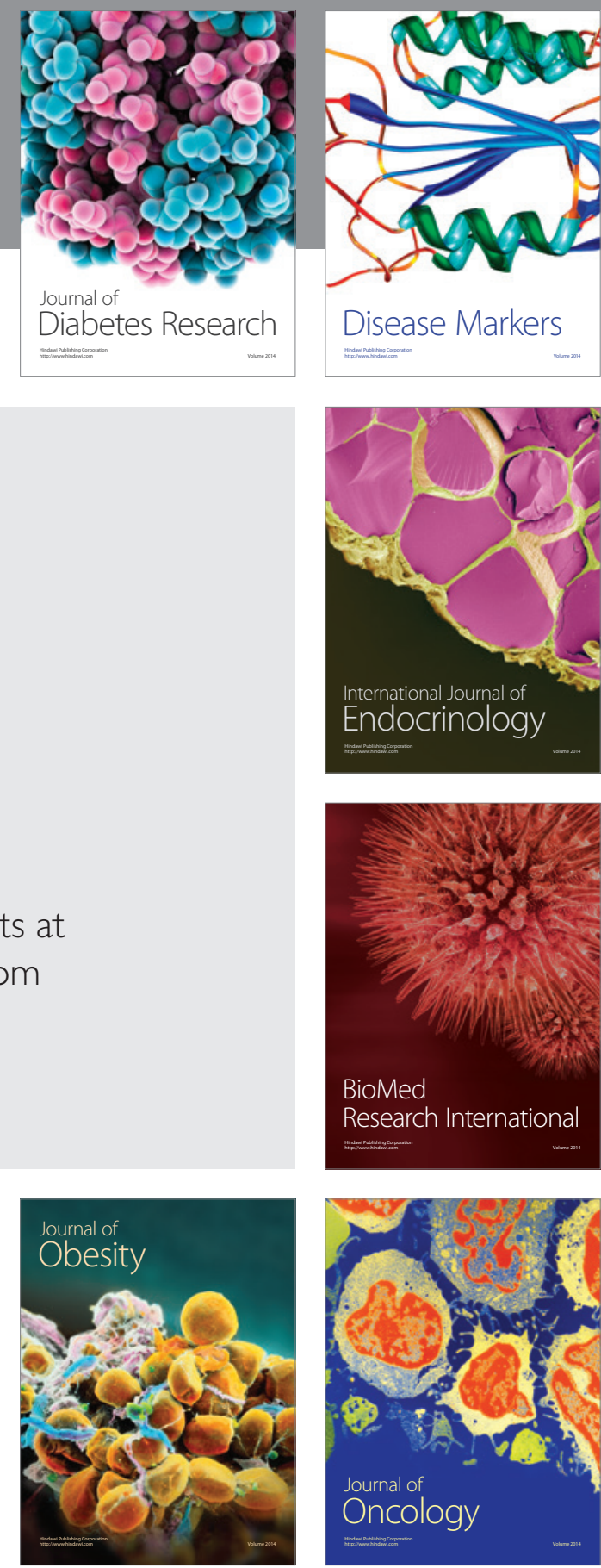

Disease Markers
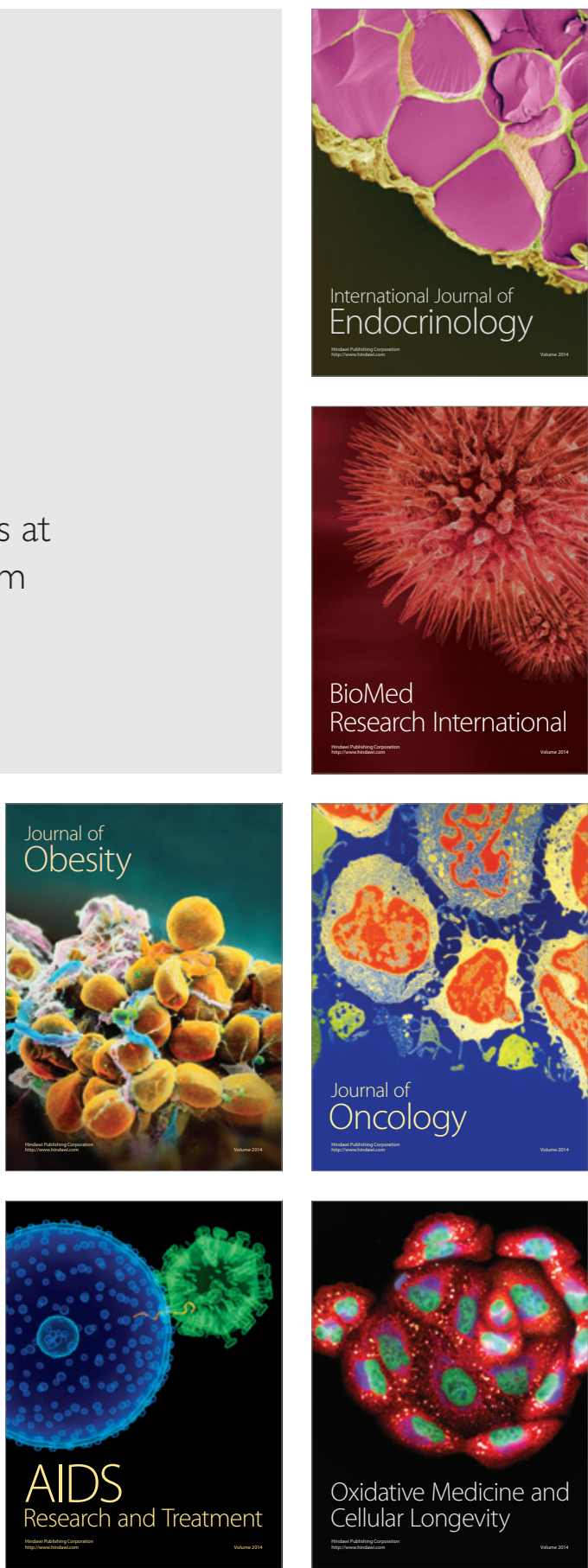